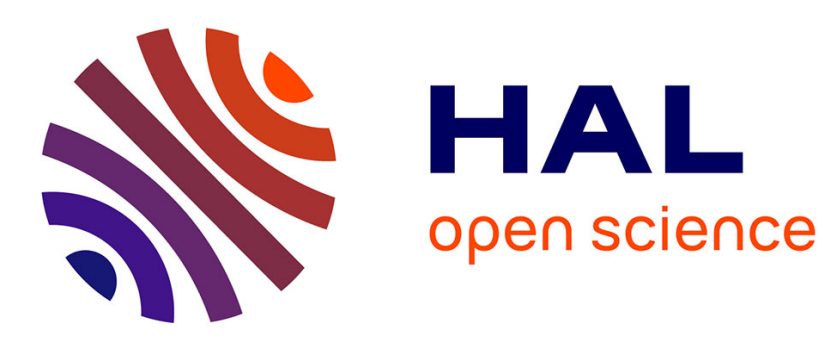

\title{
An iterative study of time independent induction effects in magnetohydrodynamics
}

Mickaël Bourgoin, Philippe Odier, Jean-François Pinton, Yanick Ricard

\section{To cite this version:}

Mickaël Bourgoin, Philippe Odier, Jean-François Pinton, Yanick Ricard. An iterative study of time independent induction effects in magnetohydrodynamics. Physics of Fluids, 2004, 16 (7), pp.25292547. 10.1063/1.1739401. hal-00492367

\section{HAL Id: hal-00492367 https://hal.science/hal-00492367}

Submitted on 15 Jun 2010

HAL is a multi-disciplinary open access archive for the deposit and dissemination of scientific research documents, whether they are published or not. The documents may come from teaching and research institutions in France or abroad, or from public or private research centers.
L'archive ouverte pluridisciplinaire HAL, est destinée au dépôt et à la diffusion de documents scientifiques de niveau recherche, publiés ou non, émanant des établissements d'enseignement et de recherche français ou étrangers, des laboratoires publics ou privés. 


\title{
An iterative study of time independent induction effects in magnetohydrodynamics
}

\author{
M. Bourgoin, P. Odier, and J.-F. Pinton ${ }^{\text {a) }}$ \\ Laboratoire de Physique, CNRS UMR\#5672, École Normale Supérieure de Lyon, 46 allée d'Italie, \\ F-69007 Lyon, France \\ Y. Ricard \\ Laboratoire des Sciences de la Terre, CNRS UMR\#5570, École Normale Supérieure de Lyon, \\ 46 allée d'Italie, F-69007 Lyon, France
}

(Received 31 October 2003; accepted 23 March 2004; published online 2 June 2004)

\begin{abstract}
We introduce a new numerical approach to study magnetic induction in flows of an electrically conducting fluid submitted to an external applied field $\mathbf{B}_{0}$. In our procedure the induction equation is solved iteratively in successive orders of the magnetic Reynolds number Rm. All electrical quantities such as potential, currents, and fields are computed explicitly with real boundary conditions. We validate our approach on the well known case of the expulsion of magnetic field lines from large scale eddies. We then apply our technique to the study of the induction mechanisms in the von Kármán flows generated in the gap between coaxial rotating disks. We demonstrate how the omega and alpha effects develop in this flow, and how they could cooperate to generate a dynamo in this homogeneous geometry. We also discuss induction effects that specifically result from boundary conditions. (C) 2004 American Institute of Physics. [DOI: 10.1063/1.1739401]
\end{abstract}

\section{INTRODUCTION}

When a magnetic field is applied to a flow of an electrically conducting fluid, complex induction mechanisms occur and induced currents and magnetic field are generated. For certain particular flows, this induction process may generate an instability where the induced magnetic field adds up to the initial one such that a large-scale field can grow. This phenomenon, known as the magnetohydrodynamic (MHD) dynamo instability, is thought to be the source of cosmic bodies' magnetic fields, as originally suggested by Larmor in 1919. ${ }^{1}$

Experimentally, dynamo action has first been produced by the controlled motion of solid metal rotors. The setup designed by Lowes and Wilkinson ${ }^{2}$ uses two metal cylinders with their axis at an angle. The differential rotation generated by each rotating cylinder inside a stationary conductor converts a poloidal field into an azimuthal one. A loop-back instability mechanism is created because the azimuthal induced field produced by each cylinder plays the role of an axial field for the other rotating conductor. Fluid dynamos have also been demonstrated experimentally by the Karlsruhe and Riga experiments., ${ }^{3,4}$ These experiments have been built so that the mean fluid flow mimics model (laminar) configurations where dynamo action has been analytically calculated." ${ }^{5,6}$ In each case, observations have shown that the experimental dynamo onset is very close to that calculated from the laminar mean flow alone. ${ }^{7,8}$ In order to study the backreaction and the time dynamics of fluid dynamos above threshold, it would be desirable to build less con-

\footnotetext{
a) Author to whom correspondence should be addressed. Electronic mail: pinton@ens-lyon.fr
}

strained flows that are capable of self-generation. In this quest, several groups have focused on swirling flows generated by the rotation of coaxial impellers in a closed volume. 9 These flows possess differential rotation and helicity, two ingredients that play a central role in dynamo self-generation. ${ }^{10}$ Kinematic simulations have shown that dynamo action is a possibility in these flows, ${ }^{11,12}$ but a dynamo loop-back mechanism has been clearly identified, as it was for instance for the Lowes and Wilkinson dynamo.

The understanding of this mechanism is of crucial importance for experimentalists. Indeed, kinematic simulations show that for any experimental configuration, the critical value for the control parameter (magnetic Reynolds number, $\mathrm{Rm}$, see next section) is always very close to the maximum value achievable in the experiment. If one wants to increase the magnetic Reynolds number, a strong limiting factor is the cost in power consumption $P$ of the engines driving the flow which scales like $\mathrm{Rm}^{3} .{ }^{15,19}$ The success of an experimental dynamo relies therefore on a proper identification of the loop-back mechanism and of the geometry of the magnetic field and electrical currents in the experimental vessel to optimize the design of the experiment.

The purpose of this paper is to study in detail the induction mechanisms that occur in von Kármán (VK) swirling flows, generated inside a cylinder by the rotation of one or two coaxial disks. However, the method could be easily extended to other types of geometries, some of which more appropriate for geophysical applications.

We consider the induced magnetic and electrical response of the flow when an external field is applied. Traditional techniques to solve the equation governing the behavior of the magnetic field in a fluid [Eq. (1), Sec. II A] use a decomposition on special functions and express boundary 
conditions as nonlocal spectral conditions, therefore only allowing the treatment of simple boundary geometries, such as spherical or cylindrical vessels. We propose here a quasistatic perturbative approach in which complex boundary conditions (close to experimental reality) can be conveniently implemented. The net magnetic induction is expressed as the result of an iterative process where the flow subjected to a given field of order $\mathbf{B}_{k}$ induces the next order $\mathbf{B}_{k+1}$. For each iterative step we compute all electromagnetic quantities involved in the induction process: induced electromotive force (e.m.f.), currents, and magnetic field. In this approach successive iterations correspond to the onset of new couplings as $\mathrm{Rm}$ increases. An experimentalist can therefore identify these couplings and understand how they cooperate to favor or hinder the dynamo action and also understand the role of the boundary conditions (see Figs. 9, 10, and 13). This provides a useful guidance for the design and optimization of experiments. Actually, the work described in this paper was originally motivated by the necessity for experimentalists to better understand the path of electrical currents, without which, for instance, the effects related to the boundary conditions (see Fig. 13) cannot be understood.

The paper is organized as follows: in Sec. II, we present in detail our iterative approach, its links with more traditional kinematic simulations, the implementation of boundary conditions and numerical strategies. In Sec. III, we revisit the process of expulsion of magnetic field lines by a large eddy as a test case for the iterative method. We then consider the induction due to differential rotation (Sec. IV) and helical motion (Sec. V) in VK flows. In Sec. VI, we discuss the possible generation of an $\alpha-\omega$ dynamo in this geometry. Section VII is devoted to the study of an induction mechanism in VK geometry that is mainly due to the boundary conditions at the lateral wall of the flow.

\section{A QUASISTATIC ITERATIVE APPROACH}

Our aim is to describe and understand the induction effects that take place in a stationary flow of an electrically conducting fluid submitted to an external magnetic field, when the magnetic Reynolds number is increased ( $\mathrm{Rm}$ is defined as the ratio of induction to Joule dissipation effects). The iterative procedure consists in solving step by step the induction equation, and obtain the induced field as a series in $\mathrm{Rm}$. One computes to first order in $\mathrm{Rm}$ the magnetic field $\mathbf{B}_{1}$ induced from the applied field $\mathbf{B}_{0}$; the procedure is repeated to compute the field $\mathbf{B}_{2}$ induced from $\mathbf{B}_{1}$ at first order, and so forth. Since the induction equation is linear, the net magnetic field is the sum over all contributions. As we will see, this approach converges strictly only at small Rm, but it can be extended to larger values and the results are in remarkable agreement with experimental data.

\section{A. Induction equation and boundary conditions}

In the MHD approximation, ${ }^{10}$ the magnetic response of a flow with velocity $\mathbf{u}(\mathbf{r})$ to an applied uniform field $\mathbf{B}_{0}$ is governed by

$$
\partial_{t} \mathbf{B}=\nabla \times\left[\mathbf{u} \times\left(\mathbf{B}+\mathbf{B}_{0}\right)\right]+\lambda \Delta \mathbf{B},
$$

$$
\boldsymbol{\nabla} \cdot \mathbf{B}=0,
$$

where $\lambda=1 / \mu_{0} \sigma$ is the fluid magnetic diffusivity (electrical conductivity $\sigma)$. The flow velocity $\mathbf{u}(\mathbf{r})$ is assumed to be stationary. MHD experiments being usually conducted in liquid metals, we also assume that the flow is incompressible, $\boldsymbol{\nabla} \cdot \mathbf{u}=0$. We further assume that Lorentz forces remain small compared to inertial and pressure forces, i.e., the magnetic field never grows strong enough to perturb the prescribed hydrodynamic velocity field-the interaction parameter remains small, $N=\sigma L B_{0}^{2} / \rho U$ where $\rho$ is the fluid's density, and $U, L$ characteristic velocity and size of the flow. The problem considered here is of the same nature as addressed by kinematic simulations in which the flow is fixed and one studies its magnetic response.

The induction Eq. (1) must be supplemented with boundary conditions. Their choice depends on the definition of the MHD "system." One simple and elegant solution is to consider the system as being the unbounded space, in which case the condition is that of vanishing magnetic field at infinity (Dirichlet). In this case, the inhomogeneities of electrical conductivity are taken into account as an additional term in the induction equation

$$
\partial_{t} \mathbf{B}=\nabla\left[\mathbf{u} \times\left(\mathbf{B}+\mathbf{B}_{0}\right)\right]+\lambda \Delta \mathbf{B}+(\boldsymbol{\nabla} \times \mathbf{B}) \times \nabla \lambda .
$$

This formulation yields a well posed problem, although not practical for numerical implementations. One thus reverts to a finite homogeneous system with specific conditions at the flow walls: in the case of insulating outer walls, Eq. (1) is then supplemented by the condition of continuity of the magnetic field at the wall (absence of outgoing currents).

\section{B. The iterative scheme}

In order to compute the induced magnetic field for a given applied field, conventional techniques as used in kinematic simulations would directly solves Eq. (1). This would yield a complete solution, including its time dependence. However, we are interested in understanding how the system reaches a steady-state equilibrium between diffusion and induction, a process that we call an "induction mechanism." We wish to analyze the role of the various components of the velocity field and their gradients, and the role of the boundary conditions. We thus develop an approach in which successive contributions to the net induction (linear in Rm, then quadratic, cubic, etc.) are individually identified and their relative importance estimated as $\mathrm{Rm}$ is varied.

For a given velocity distribution $\mathbf{u}(\mathbf{r})$ and applied field $\mathbf{B}_{0}$, we search for steady solutions of the induction equation

$$
\mathbf{B}=\mathbf{B}_{0}+\mathbf{B}_{\text {ind }}, \quad \mathbf{B}_{\text {ind }}=\sum_{k=1}^{\infty} \mathbf{B}_{k}, \quad\left|\mathbf{B}_{k}\right| \sim \mathcal{O}\left(\mathrm{Rm}^{k}\right) .
$$

In the numerical computation the magnetic Reynolds number $\mathrm{Rm}$ is defined as

$$
\mathrm{Rm}=U L / \lambda,
$$

where $U=\max _{\mathbf{r}}(|\mathbf{u}(\mathbf{r})|)$ and $L$ are characteristic integral velocity and length scales of the flow (for example $L$ can be the radius $R$ of the cylinder in VK flows). As we look for sta- 
tionary solutions, contributions at each order are obtained as solutions of a hierarchy of Poisson equations

$$
\Delta \mathbf{B}_{k+1}=-\operatorname{Rm} \nabla \times\left(\mathbf{u} \times \mathbf{B}_{k}\right),
$$

in which lengths, velocities, and magnetic fields have been nondimensionalized, respectively, by, $L, U$, and $B_{0}$.

Each step described by Eq. (6) can be interpreted as usual in terms of distorsion and transport of the magnetic field lines by the velocity gradients of the flow. Even without resolving numerically this set of equations, one has here a way, if the magnetic Reynolds number remains moderate, to picture the mechanisms involved in the induction of magnetic field, in relation with the main velocity gradients of the flow, as well as a good insight on the spatial structure of the magnetic field.

\section{Calculation at each order}

Solving Eq. (6) by a simple Poisson solver is not trivial as it would imply writing the boundary conditions for the magnetic field at the surface of a cylinder. Instead we start from electric potential and induced currents. The fields at each order are thus considered successively, using the following sequence.

(1) The electromotive force (e.m.f. in units of $U B_{0}$ ) induced by the flow motion is computed as

$$
\mathbf{e}_{k+1}=\mathbf{u} \times \mathbf{B}_{k} .
$$

(2) Since the electrical current, given by Ohm's law $\mathbf{j}=\sigma(-\boldsymbol{\nabla} \phi+\mathbf{e})$, is divergence free, the electric potential is obtained as a solution the Poisson equation

$$
\Delta \phi_{k+1}=\nabla \cdot\left(\mathbf{u} \times \mathbf{B}_{k}\right) \text {. }
$$

In the case of an insulating boundary, the condition of vanishing outgoing currents can then be written as $\mathbf{n} \cdot \nabla \phi$ $=\mathbf{n} \cdot(\mathbf{u} \times \mathbf{B})$. The earlier Poisson equation can then easily be solved as a von Neuman problem, for any kind of geometry. Note that in real flows the hydrodynamic boundary layer is very small since the magnetic Prandtl number of liquid metals is less than $10^{-5}$; as a result the viscous sublayer is of negligible extend compared to magnetic scales. A free slip rather than no-slip boundary condition for the velocity is appropriate and the boundary condition at the wall, $\mathbf{n} \cdot \boldsymbol{\nabla} \phi$ is therefore nonzero.

(3) The induced currents (in units of $\sigma U B_{0}$ ) are then computed as

$$
\mathbf{j}_{k+1}=-\nabla \phi_{k+1}+\mathbf{e}_{k+1} .
$$

(4) Finally, the magnetic field can be computed from BiotSavart formula

$$
\mathbf{B}_{k+1}(\mathbf{r})=\frac{\mathrm{Rm}}{4 \pi} \int d^{3} r^{\prime} \frac{\mathbf{j}_{k+1}\left(\mathbf{r}^{\prime}\right) \times\left(\mathbf{r}-\mathbf{r}^{\prime}\right)}{\left|\mathbf{r}-\mathbf{r}^{\prime}\right|^{3}} .
$$

In practice, the integral is only computed at the boundary and used to provide boundary conditions to solve the Dirichlet problem $\Delta \mathbf{B}_{k+1}=\operatorname{Rm} \nabla \times \mathbf{j}_{k+1}$.
(5) The magnetic Reynolds number only enters in the final step, where all contributions are collected and the fields are obtained as integer series

$$
\phi_{k}(\mathrm{Rm})=\phi_{k}(1) \mathrm{Rm}^{k-1} \Rightarrow \phi(\mathrm{Rm})=\sum_{k} \phi_{k}(1) \mathrm{Rm}^{k-1}
$$

$$
\begin{aligned}
& \mathbf{j}_{k}(\mathrm{Rm})=\mathbf{j}_{k}(1) \mathrm{Rm}^{k-1} \Rightarrow \mathbf{j}(\mathrm{Rm})=\sum_{k} \mathbf{j}_{k}(1) \mathrm{Rm}^{k-1}, \\
& \mathbf{B}_{k}(\mathrm{Rm})=\mathbf{B}_{k}(1) \mathrm{Rm}^{k} \Rightarrow \mathbf{B}(\mathrm{Rm})=\sum_{k} \mathbf{B}_{k}(1) \mathrm{Rm}^{k} .
\end{aligned}
$$

This approach is valid when the series converges, that is for a low enough value of $\mathrm{Rm}(\mathrm{Rm}<\mathrm{Rm} *)$. Numerically we observe that $\mathrm{Rm}^{*} \sim 5-30$ in the cases considered in our study. This range covers a significant fraction of magnetic Reynolds numbers that have been explored in experiments using liquid gallium ${ }^{14,15}$ and liquid sodium. ${ }^{16,18,19}$ One may also recall that the integral magnetic Reynolds number (i.e., defined on large scale physical parameters) is an upper limit for the actual magnetic Reynolds number of the flow (as a number which actually measures the strength of induction effects). ${ }^{20}$ For higher magnetic Reynolds number values, one needs to extrapolate the series expansion outside its strict convergence radius. We use Padé approximants, ${ }^{21}$ which has become traditional in these problems (see, for example, Ref. 22). It yields strikingly good results when compared to analytical solutions or measurements, as will be shown in the next sections.

\section{Numerical implementation}

As explained, our study concentrates on the von Kármán flows, although the approach could be easily applied to other geometries. In experimental configurations, von Kármán flows are generated inside a cylinder by the rotation of one or two discs whose axis of rotation coincides with the axis of the cylinder. Two regimes are of particular interest.

(1) Single disk (SD): a single disk is rotated at frequency $\Omega$, the other being held at rest. In this case the timeaveraged flow is strongly helical: it has a toroidal component, and a recirculation poloidal loop, created by the pumping effect towards the center of the rotating disc. In Duddley and James terminology, ${ }^{11}$ it is a $s_{1} t_{1}$-like flow.

(2) Double disk (DD): the disks are counter-rotated at equal rates $\Omega$. In this case, the mean flow is made of two cells with opposite toroidal velocities and two recirculation loops, with a strong shear zone in-between. Using again Duddley and James terminology, ${ }^{11}$ it is a $s_{2} t_{2}$-like flow.

In our numerical studies the flow is a synthetic velocity field that mimics the main properties of the experimental mean flows. The aspect ratio of the flow is one, i.e., the height of the cylinder is equal to its diameter (and will remain so throughout our study). We choose simple harmonic functions for the radial and azimuthal velocities $u_{r}$ and 
$u_{\theta}$ and deduce the vertical component $u_{z}$ from the incompressibility condition. For the (SD) case we use

$$
\begin{aligned}
& u_{r}(r, z)=-\sin \pi r \cos \frac{\pi(z+1)}{2}, \\
& u_{\theta}(r, z)=2(P / T)^{-1} \sin \pi r, \\
& u_{z}(r, z)=\frac{1}{\pi r} \sin \frac{\pi(z+1)}{2}(\pi r \cos \pi r+\sin \pi r),
\end{aligned}
$$

where $(r, \theta, z)$ are the cylindrical polar coordinates, and $P / T$ the ratio of the amplitude of poloidal to toroidal speeds. For the counter-rotating (DD) flow configuration the velocity field is

$$
\begin{aligned}
& u_{r}(r, z)=-\sin \pi r \cos \pi z, \\
& u_{\theta}(r, z)=2(P / T)^{-1} \sin \frac{\pi z}{2} \sin \pi r, \\
& u_{z}(r, z)=\frac{1}{\pi r} \sin \pi z(\pi r \cos \pi r+\sin \pi r) .
\end{aligned}
$$

In sharp contrast with experimental works, ${ }^{18}$ the flows studied here are smooth and laminar. The effect of turbulent fluctuations (always present in real liquid metal flows at finite $\mathrm{Rm}$ ) cannot be directly computed with this numerical scheme, and other approaches are needed. ${ }^{23}$

Figure 1(a) shows the synthetic flow profile in the (SD) case. One observes poloidal recirculation loops (arrows) as the fluid is drawn to the disk near the axis of the cylinder and ejected towards the lateral wall at the disk outer rim. The toroidal velocity is close to solid body rotation up to $r$ $\simeq 0.5$. The main motion of the flow is helical. The (DD) flow is shown in Fig. 1(b). Two poloidal recirculating loops are now created, since in each half of the cylinder the fluid near the axis is drawn to the closer disk. The toroidal motions are also of opposite directions, so that helicities add up in each half of the cylinder. In addition, there is a strong differential rotation and a shear layer/stagnation point in the middle of the cylinder. In both cases, the velocity boundary condition is that of no outgoing flow, with free slip at the surface. This latter condition is justified because the thickness of the hydrodynamic boundary layer in liquid metal flows is very small. The ratio $P / T$ of the intensity of the poloidal to toroidal velocities is adjustable in the synthetic flows; it is set to 0.8 in the examples shown in Fig. 1, and in most studies here.

Numerically, the crucial part of the scheme lies in the solution of the Poisson equations for the electric potential and for the magnetic field inside the volume. They are then solved using standard programs in the Overture ${ }^{24}$ library using the mixed Cartesian-cylindrical grid shown in Fig. 1(c).

In order to reproduce induction effects that do occur in experiments, we have also used in Sec. VII velocity fields obtained by time averaging local velocity measurements in laboratory flows. These fields have been provided by our colleagues at Commissariat à l'Energie Atomique (CEA) in Saclay, from measurements in a VK flow in water. ${ }^{25}$

\section{E. Iterative approach and dynamo action}

Induction computed with the iterative approach can be linked to dynamo self-generation as follows. Let us rewrite the magnetic field induced at order $k+1$ from the field at order $k$ as

$$
\mathbf{B}_{k+1}=-\operatorname{Rm} \Delta^{-1}\left[\boldsymbol{\nabla} \times\left(\mathbf{u} \times \mathbf{B}_{k}\right)\right]=\operatorname{Rm} \mathcal{G}\left(\mathbf{B}_{k}\right),
$$

where we introduce the operator $\mathcal{G}$ :

$$
\mathcal{G} \bullet=\Delta^{-1}[\nabla \times(\mathbf{u} \times \bullet)] .
$$

Let us assume further that an induction loop back mechanism may be identified for a given velocity field and geometry, i.e., that there exists a magnetic field distribution at order $k, \mathbf{B}_{k}$, such that after $n$ induction iterations, the resulting magnetic field is similar in geometry with the initial $\mathbf{B}_{k}$ :

$$
\mathbf{B}_{k+n}(\mathrm{Rm})=\operatorname{Rm}^{n}(\mathcal{G})^{n}\left(\mathbf{B}_{k}\right)=\gamma \operatorname{Rm}^{n} \mathbf{B}_{k},
$$

with $\gamma$ a constant factor that depends on the flow and on the initial magnetic field geometry. At a given magnetic Reynolds number the net amplification factor is thus $\gamma \mathrm{Rm}^{n}$ [i.e., $\gamma \mathrm{Rm}^{n}$ and $\mathbf{B}$ are eigen value and eigen vector of the operation $\left.(\mathcal{G})^{n}\right]$. Positive $\gamma$ values lead to a growth of the magnetic field mode $\mathbf{B}_{k}$ and thus may lead to a dynamo instability if $\gamma \mathrm{Rm}^{n}>1$, the critical magnetic Reynolds number being

$$
\mathrm{Rm}^{c}=\gamma^{-n}
$$

(note again that only stationary dynamos can be captured using this procedure). Negative values of $\gamma$ correspond to processes where the induced magnetic field opposes the applied one. Such situation occur for example in the expulsion of magnetic field lines from eddies ${ }^{26,27}$ - this case will be discussed in detail in Sec. III.

When a positive loop-back mechanism exists, the geometry of the marginal mode can also be identified, in much the same manner as in kinematic dynamo studies. Indeed, in kinematic approaches, the neutral mode satisfies

$$
\mathcal{N}_{\operatorname{Rm}^{c}}\left(\mathbf{B}_{\text {marginal }}\right)=0,
$$

with $\mathrm{Rm}^{c}$ the critical magnetic Reynolds number, and the operator $\mathcal{N}_{\mathrm{Rm}}$ defined as

$$
\mathcal{N}_{\mathrm{Rm}} \bullet=\operatorname{Rm} \boldsymbol{\nabla} \times(\mathbf{u}(\mathbf{r}) \times \bullet)+\Delta \cdot .
$$

Comparing the definition of the operators $\mathcal{G}$ and $\mathcal{N}_{\mathrm{Rm}}$ [Eqs. (17) and (21)], Eq. (20) can be interpreted as an $n$ $=1$ loop-back induction

$$
\mathbf{B}_{\text {marginal }}=\operatorname{Rm}^{c} \mathcal{G}\left(\mathbf{B}_{\text {marginal }}\right)=\operatorname{Rm}^{c} \gamma \mathbf{B}_{\text {marginal }},
$$

with again the condition $\operatorname{Rm}^{c} \gamma=1$. If the iteration procedure has identified a positive loop-back mechanism in $n$ steps, $\left(\mathbf{B}_{0}, \mathbf{B}_{1}, \ldots, \mathbf{B}_{n}\right)$, one can easily show that there exists a linear combination $\mathbf{B}_{\text {marginal }}=\Sigma_{k=0 \ldots n-1} \lambda_{k} \mathbf{B}_{k}$ such that $\mathcal{G} \mathbf{B}_{\text {marginal }}$ $=\gamma \mathbf{B}_{\text {marginal }}$ (in fact $\mathbf{B}_{\text {marginal }}$ is an eigenvector of the subspace stable under $\mathcal{G}$ ). The neutral mode is thus obtained from the structure of the magnetic field induced at each step in a loop-back mechanism. The occurrence of such processes and its applications to dynamo action in VK flows will be discussed in Sec. VI. 

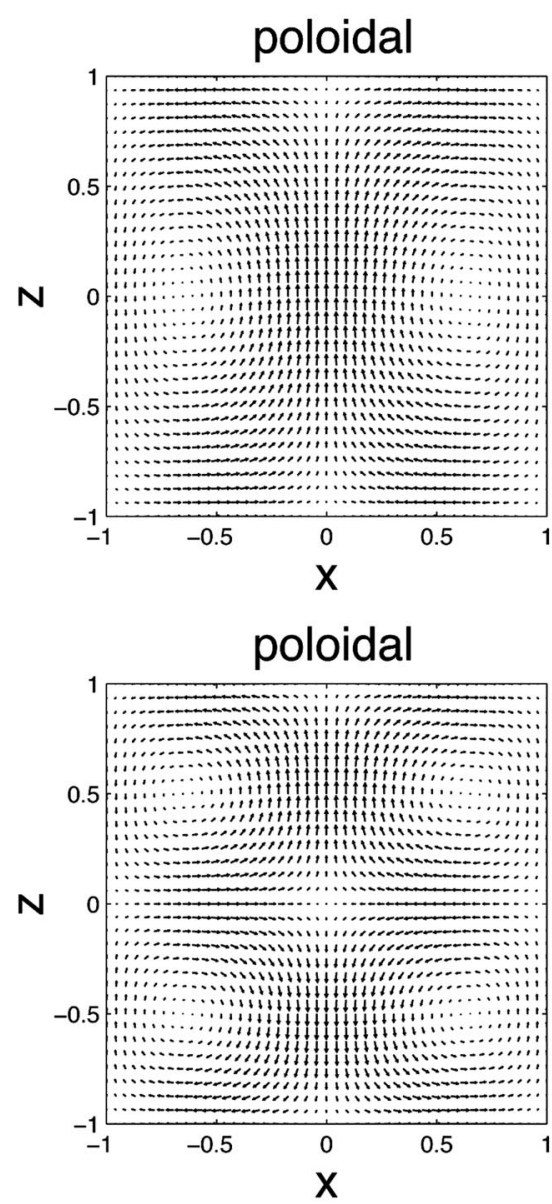

(b)

(a)
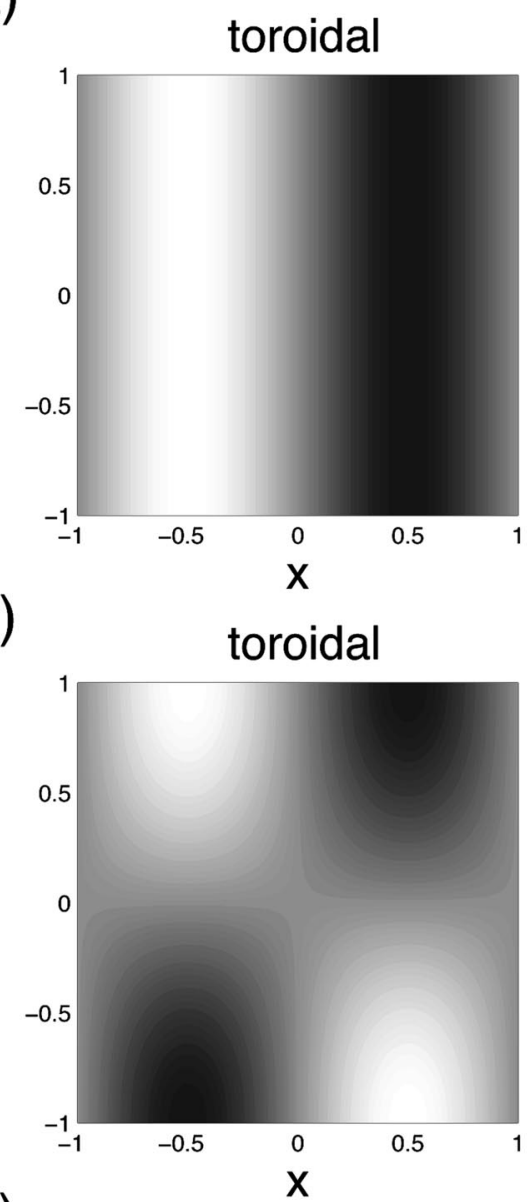

(c)

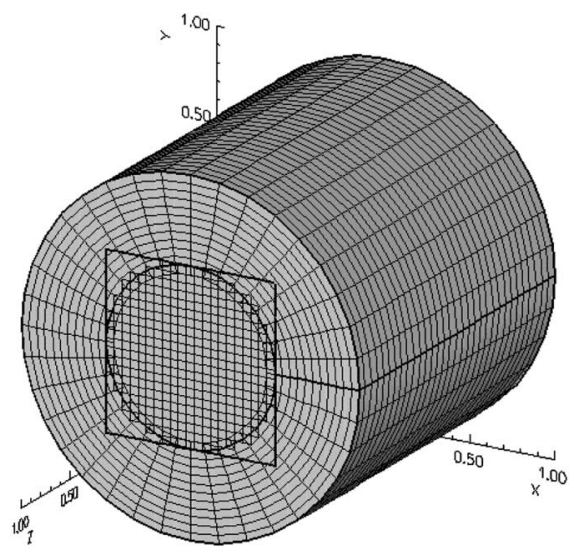

\section{COROTATING FLOW: EXPULSION BY LARGE SCALE EDDIES}

We first apply the iterative approach to a case that has been well studied analytically ${ }^{28}$ and experimentally: ${ }^{27}$ the expulsion of magnetic field lines by large eddies. To this end, the computational cylinder is divided into a region in solid body rotation $\left(u_{r}=u_{z}=0, u_{\theta}=\Omega r\right.$, up to radius $\left.R=1\right)$ surrounded by a region at rest (up to $R=2$ ); the medium within the cylinder has uniform electrical conductivity $\sigma$ and is surrounded by insulating material. The cylinder is periodized in the $z$ direction, so the system is quasi-two-dimensional. An external magnetic field $\mathbf{B}_{0}=B_{0} \hat{x}$ is applied perpendicular to the cylinder axis, along the $x$ axis. The solution to this problem can be found analytically. ${ }^{28}$ In cylindrical polar coordinates, one has for the stationary state

$$
\begin{aligned}
& B_{r} / B_{0}=r^{-1}\{\mathcal{R}[f(r, p) \cos \theta]-\mathcal{I}[f(r, p) \sin \theta]\}, \\
& B_{\theta} / B_{0}=-\partial_{r} \mathcal{I}[f(r, p)],
\end{aligned}
$$



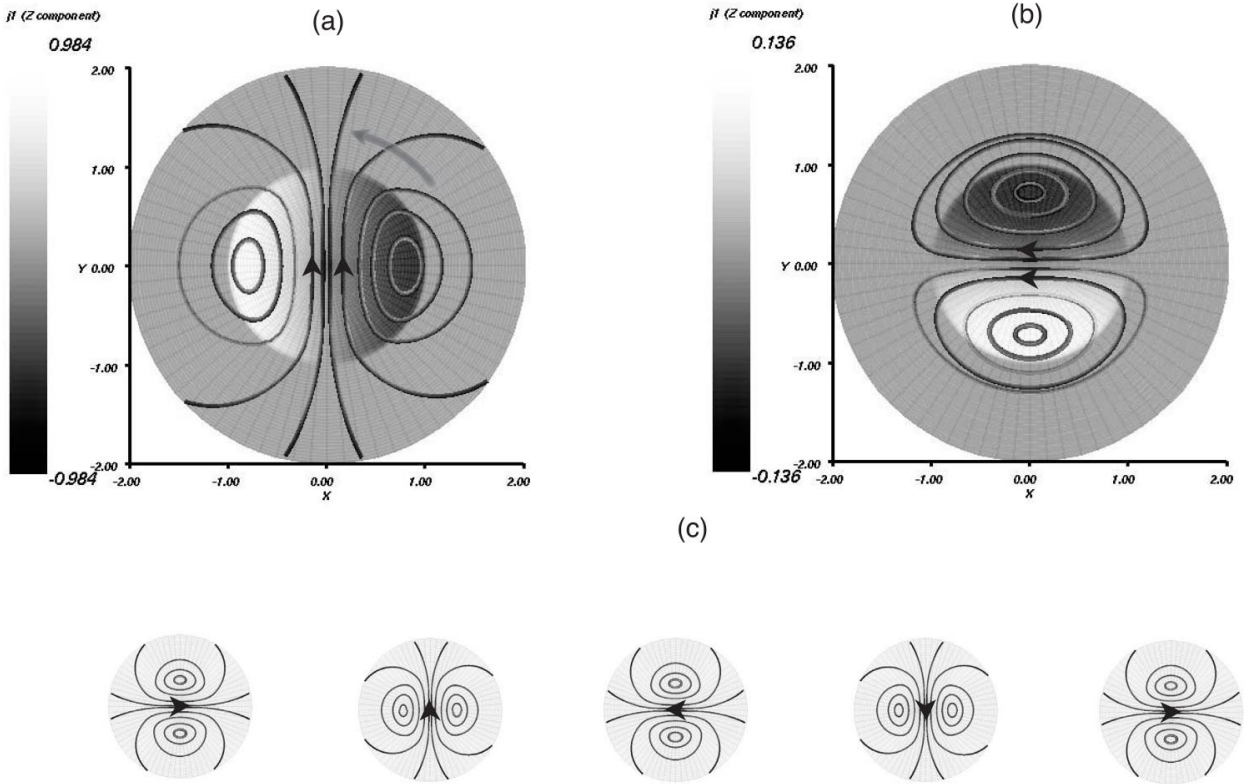

FIG. 2. Expulsion by rotation. (a) First order induction; currents are shown as a gray scale, while the lines give selected magnetic field paths; note that a domain of radius $2 R$, twice the cylinder size, is represented. (b) Idem for second order induction; (c) iteration steps of order 20-24, left to right.

$$
\begin{aligned}
& f(p, r)=D J_{1}(p r), \quad r<R, \quad f(r, p)=r+C / r, r>R \\
& p=\frac{(i-1) \sqrt{\mathrm{Rm}}}{\sqrt{2} R}, \quad D=\frac{2}{p J_{0}(p R)} \\
& C=\frac{2 R J_{1}(p r)-p R^{2} J_{0}(p r)}{p J_{0}(p r)}
\end{aligned}
$$

$\left[\mathcal{R}\right.$ and $\mathcal{I}$ real and imaginary parts and $J_{n}(x)$ are Bessel functions]. Physically, the initially transverse field lines are advected and stretched by the solid body rotation, thus "being pushed away" to the sides of the cylinder while the inner core field decreases under diffusion. From an equivalent point of view, the applied field is time periodic in the reference frame of the rotating core and thus can penetrate only as far as the skin depth $\delta=R / \sqrt{\mathrm{Rm}}$.

We now show how this behavior is described by the iterative approach. At first order the induced e.m.f.

$$
\mathbf{e}_{1}=\mathbf{u} \times \mathbf{B}_{0}=-r \Omega B_{0} \cos \theta \hat{z}=-x \Omega B_{0} \hat{z}
$$

is purely axial and divergence-free. As a result the induced current density $\mathbf{j}_{1}$ has no potential contribution and is identical to the e.m.f. distribution [see Eq. (9)]. It is thus made of axial currents that flow in opposite directions on each side of the $y O z$ plane, as shown in Fig. 2(a). The resulting induced magnetic field at first order $\mathbf{B}_{1}$ has a dipole structure, aligned with the $y$ axis: the main effect of the rotation is to induce a field component perpendicular to the applied one. The second order induction just repeats this sequence: as $\mathbf{B}_{1}$ lies in the $(x, y)$ plane, the second order electric potential is divergence free and the second order currents are $\mathbf{j}_{2}=\mathbf{e}_{2}$ $=\mathbf{u} \times \mathbf{B}_{1}$. They flow axially in opposite direction on either side of the $x O z$ plane-Fig. 2(b). The resulting induced magnetic field is a dipole along the $x$ axis and opposite to $\mathbf{B}_{0}$. This two-step process thus tends to reduce the applied field, it is the essential mechanism of the expulsion of the magnetic field from the core of the rotating motion-as an illustration, the induction steps 20-24 are displayed in Fig. 2(c) showing the stability of the order-two cycle of induction patterns. In fact the two modes, " $x$-axis dipole" $\mathbf{b}_{1}$ and " $y$ axis dipole" $\mathbf{b}_{2}$, form a stable subspace under $\mathcal{G}$, such that

$$
\begin{array}{ll}
\mathcal{G}\left(\mathbf{b}_{1}\right)=\gamma_{1} \mathbf{b}_{2}, & \gamma_{1}=1.697 \times 10^{-1}, \\
\mathcal{G}\left(\mathbf{b}_{2}\right)=\gamma_{2} \mathbf{b}_{1}, & \gamma_{2}=-1.697 \times 10^{-1}, \\
\mathcal{G}^{2}\left(\mathbf{b}_{1,2}\right)=\gamma \mathbf{b}_{1,2}, & \gamma=\gamma_{1} \gamma_{2}=-2.879 \times 10^{-3} .
\end{array}
$$

The negative value of $\gamma$ leads to the expulsion process. We show in Figs. 3(a) and 3(b) the profile of magnetic field lines obtains after the iteration of 40 steps. At low magnetic Reynolds number $[\mathrm{Rm}=1$ in (a)] the raw series is computed; in (b) the Reynolds number is equal to 10, larger than the radius of convergence of the series and Padé resummation has been used to ensure convergence. The results are in very good agreement with the original numerical calculations by Weiss. ${ }^{26}$ To be more quantitative, we plot a comparison of the numerical and analytical variations with Rm in Figs. 3(c) and 3(d). In (c) the raw series up to order 40 is used; the series diverges for $\mathrm{Rm}>\mathrm{Rm}^{*} \simeq 5.5$, but below the agreement is excellent. If Padé resummation is applied, as in (d), one gets an excellent agreement at least up to $\mathrm{Rm} \simeq 40$. Note that the observed radius of convergence of the series is very well approximated by $\mathrm{Rm}^{*}=1 / \sqrt{\gamma_{1} \gamma_{2}}=5.89$, as one could have inferred by starting the iteration process with one of the stables modes $\left(\mathbf{b}_{1}, \mathbf{b}_{2}\right)$ : the net induced field is obtained as a series in powers of $\gamma_{1} \gamma_{2} \mathrm{Rm}^{2}$.

\section{OMEGA EFFECT AND FIELD EXPULSION}

In this section we consider the (DD) case of the VK flow, generated when counter-rotation at equal rates is 
(a)

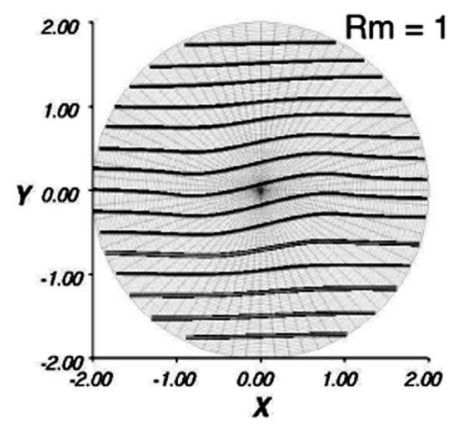

(c)

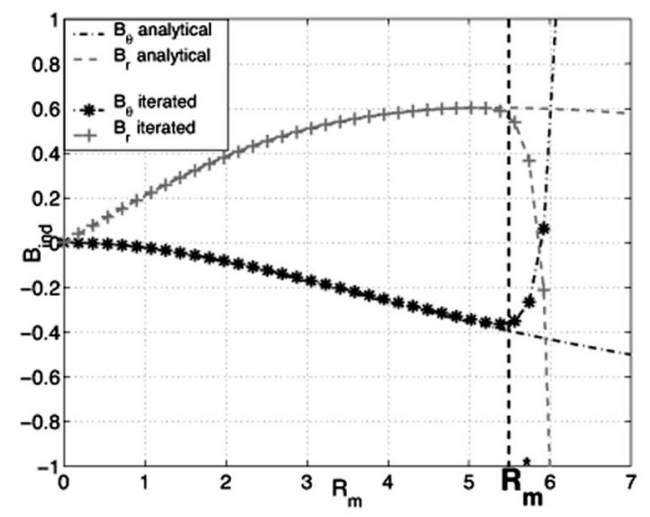

(b)

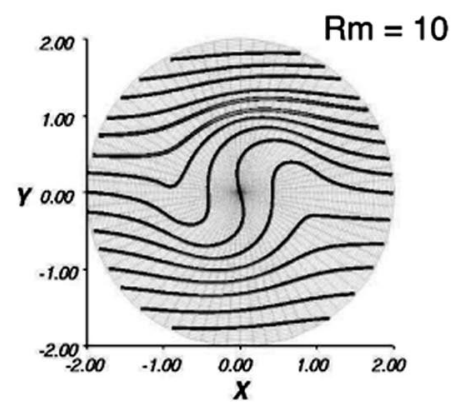

(d)

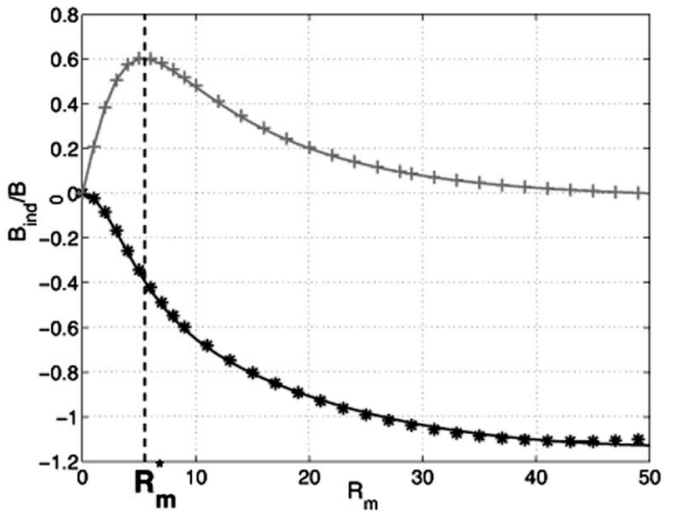

FIG. 3. Expulsion by rotation. (a) and (b) Magnetic field lines computed using the iterative approach, respectively at $\mathrm{Rm}=1$ and $\mathrm{Rm}=10$. (c) and (d) Detailed comparison of the magnetic profiles $B_{\theta}(r)$ and $B_{r}(r)$ obtained in the analytical solution and in the numerical, iterative scheme (lines with symbols). In (c), terms in series up to order 40 have been computed: the divergence of the series is clearly visible for $\mathrm{Rm}>\mathrm{Rm} *$. In (b) and (d) the Padé resummation is used.

imposed-Fig. 1(b). We consider in the section the response of the fluid to an applied field, homogenous and aligned with the cylinder axis ( $z$ direction). At low Rm, the differential rotation (velocity gradients $\partial_{z} u_{\theta}$ ) generates an azimuthal magnetic field, via the $\omega$ effect. In the poloidal recirculation loops, the velocity gradients $\partial_{z} u_{r}$ and $\partial_{z} u_{z}$ also induce axial and radial magnetic fields. At high Rm, expulsion occurs and induction decreases.

\section{A. First order induction}

In this geometry, experiments ${ }^{14}$ have shown that the magnetic induction varies linearly with $\mathrm{Rm}$ at low $\mathrm{Rm}$, so that first order calculations should give the main features. The first order e.m.f., $\mathbf{e}_{1}=\mathbf{u} \times \mathbf{B}_{0}$, gives rise to an electric potential that solves

$$
\Delta \phi_{1}=\mathbf{B}_{0} \cdot \boldsymbol{\omega},
$$

[from Eq. (8)] where $\boldsymbol{\omega}=\boldsymbol{\nabla} \times \mathbf{u}$ is the vorticity of the flow. The advantage of this notation is to emphasize the role played by vorticity in the induction process. Note that VK flows have a vorticity field that has the same topological structure as the velocity; in this sense they are very similar to Beltrami flows. Contrarily to the case considered in the previous section, the electric potential must be nonzero to ensure that currents remain divergence free. Its topology is shown in Fig. 4: it has essential contributions on the cylinder axis and near the outer rims of the ends of the cylinder. Indeed, on the axis the vorticity is aligned with the applied field, with opposite signs at each end; this leads to the axial potential difference. Near the outer rims of the cylinder, the axial vorticity is reversed compared to that on the axis; it generates in this region a radial potential difference. The induced currents result from the potential distribution and local induction $\mathbf{j}_{1}=-\boldsymbol{\nabla} \phi_{1}+\mathbf{e}_{1}$; their geometry is quite complex, but two limiting cases can be identified.

(i) One case is shown in Fig. 4(a). The axial potential difference drives an axial current (actually all axial currents are necessarily of potential origin since $\mathbf{B}_{0}$ is axial) from one end of the cylinder to the other. Then currents are transported away from the axis by the induction e.m.f. $\mathbf{u} \times \mathbf{B}_{0}$-note that this is done against the radial potential difference, explaining why the current spirals radially outward (respectively inward) in this region. When at the outer wall, the current can again flow axially under the action of the outer axial potential difference. The results is the formation of poloidal currents on a torus, which generate a toroidal magnetic field. This is the $\omega$ effect, usually described in terms of torsion of axial magnetic field lines, interpreted here in terms of electric potential and current paths.

(ii) Another limiting case corresponds to purely azimuthal currents which can only be generated from the induction e.m.f. $\mathbf{u} \times \mathbf{B}_{0}$ since the flow is axisymmetric. In the me- 
(a)

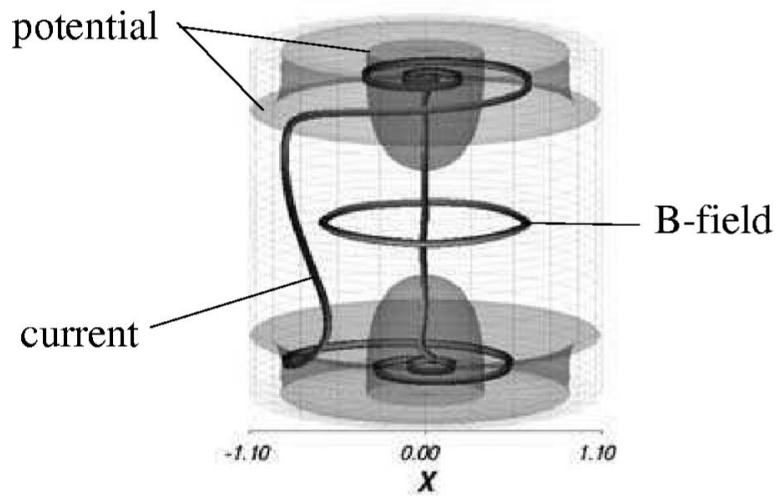

(b)

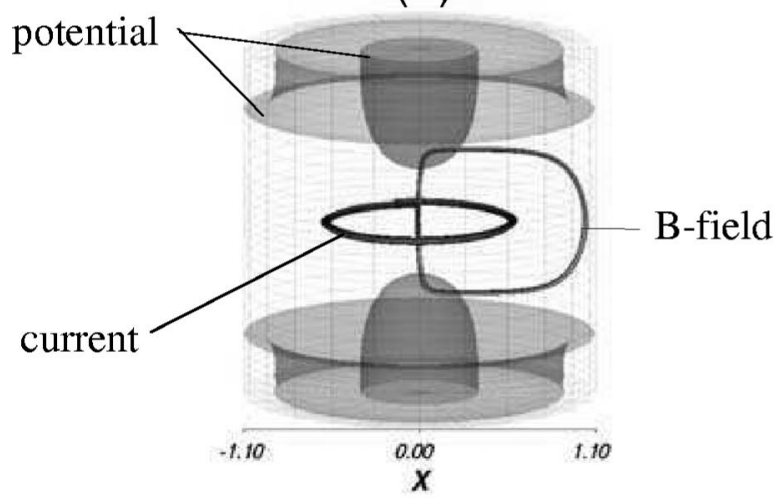

(c)

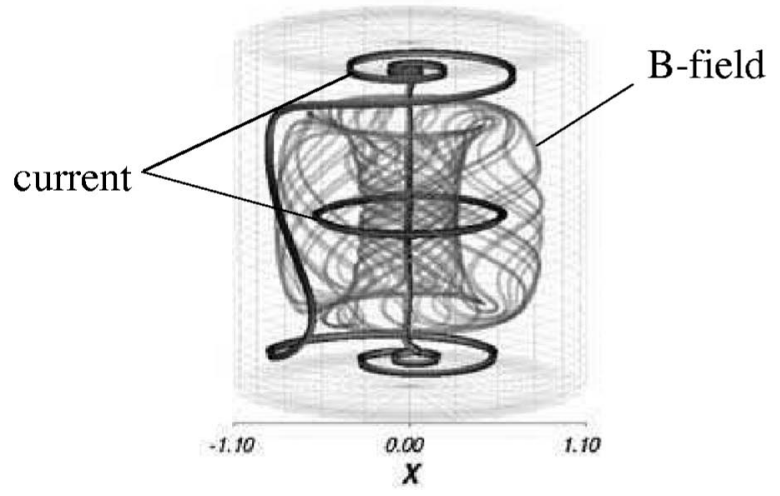

FIG. 4. Axial applied field. (a) Effect of differential rotation (toroidal flow): isopotential surfaces (their signs are opposite at top and bottom locations on the axis, and again between inner and outer radial rims - cf. text), example of induced current path and resulting induced field line; (b) effect of poloidal flow; (c) intermediate current lines.

dian plane, the radial velocity drives an azimuthal currentFig. 4(b). It the creates a poloidal magnetic field, parallel to the applied field along the axis and with the opposite direction near the cylinder outer wall. This effect corresponds to the stretching of the applied magnetic field lines by the poloidal recirculation loops of the flow.

The general current distribution induced at first order combines features of these limiting cases, see Fig. 4(c). This generates a first order magnetic field that has both toroidal and poloidal components.

\section{B. Higher orders}

At second order, one important effect is that the toroidal field induced at first order is advected by the radial flow, due to the $-u_{r} \partial_{r} B_{1, \theta}$ induction term-see Refs. 10 and 15 for a detailed discussion of the $(\mathbf{u} \cdot \nabla) \mathbf{B}$ and $(\mathbf{B} \cdot \nabla) \mathbf{u}$ induction terms that stem from Eq. (1). Since $B_{1, \theta}(r)$ in the bulk of the cylinder is maximum for $r \simeq 0.5$, the second order azimuthal field locally opposes the first order induction except near the axis of the cylinder-compare the toroidal fields in Figs. 5(a) and 5(b). The effect of the poloidal flow is thus to reduce the initial $\omega$ effect. (Another induction process yields the same result: the differential rotation acting on the axial component $B_{1, z}$ generated at first order by the poloidal velocity.) One thus expects that as the magnetic Reynolds number is increased, the efficiency of the conversion of the applied axial field into a toroidal component will decrease. This can also been viewed as the result of the progressive expulsion of the applied magnetic field by the poloidal flow: as Rm increases, the axial magnetic field concentrates along the axis of the cylinder, away from regions where the differential rotation generates efficiently a toroidal component. This behavior is indeed observed as iterations of the induction are summed into the net magnetic field. Figure 5(c) shows the evolution with $\mathrm{Rm}$ of all components of the field measured in the midplane at $r=0.5$. The induction of a toroidal component [in the figure it corresponds to $-B_{x}$ at the location $(x=0, y$ $=0.5, z=0)]$ increases up to $R m \simeq 12$, and then saturates and even decreases as $\mathrm{Rm}$ is further increased. The same phenomenon occurs for the axial component: as the applied field is progressively expelled from the poloidal recirculation loops, it can no longer be distorted by the axial velocity gradients. This is further evidenced in Fig. 5(d), where the variations with $\mathrm{Rm}$ of the axial induced field are compared for points at increasing radial distances: the axial stretching remains only in the immediate vicinity of the axis. Indeed, as $r$ gets larger than about 0.2 , the induction actually tends to oppose the applied field ( $B_{\text {ind }} \rightarrow-1$ as $\mathrm{Rm} \rightarrow \infty$ ), so that the net axial magnetic field inside the poloidal recirculation loops tends to zero.

At large orders, the dominant role of field expulsion is clearly evidenced by the fact that the iteration of the induction process yields a negative loop-back mechanisms. Steps 31-34 are shown in Fig. 6. In three steps, a magnetic field structure with a sign opposite to the starting one is obtained. Numerically, one finds $\mathbf{B}_{34} \simeq \gamma \mathbf{B}_{31}$, with $\gamma=-3.87 \times 10^{-5}$; the scalar product of the two fields, $\int d^{3} r \mathbf{B}_{31} \cdot \mathbf{B}_{34}$, is equal to -0.99 when $\mathbf{B}_{31}$ and $\mathbf{B}_{34}$ are normalized. One can thus construct from the family $\left(\mathbf{B}_{31}, \mathbf{B}_{32}, \mathbf{B}_{33}\right)$ a stable subspace and an eigenmode with a negative eigenvalue $\tilde{\gamma}=\gamma^{1 / 3}=-3.38$ $\times 10^{-2}$. This value correctly gives the radius of convergence of the integer series $\mathrm{Rm}^{*}=1 / \tilde{\gamma}=29.6$ (observed numerically, but not shown in Fig. 5 where Padé approximants have been plotted).

At a large magnetic Reynolds number, we thus observe that a mechanism as efficient as the differential rotation can ultimately be masked by the effect of expulsion by large scale eddies. The expulsion of the axial field could be lessened with a lower poloidal to toroidal ratio of characteristic 


\section{$\mathrm{B}_{1}$ poloidal}

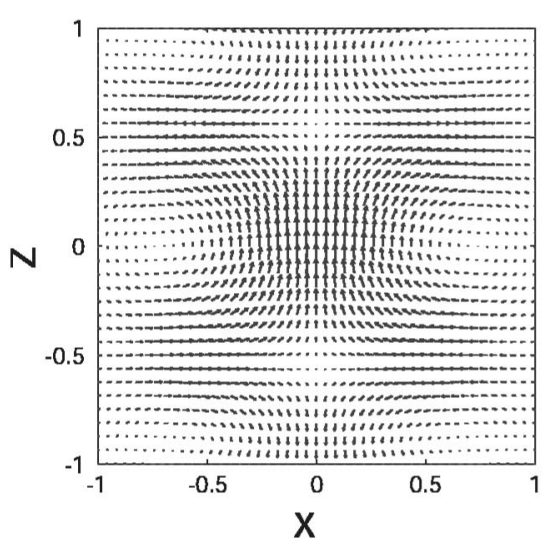

$\mathrm{B}_{2}$ poloidal

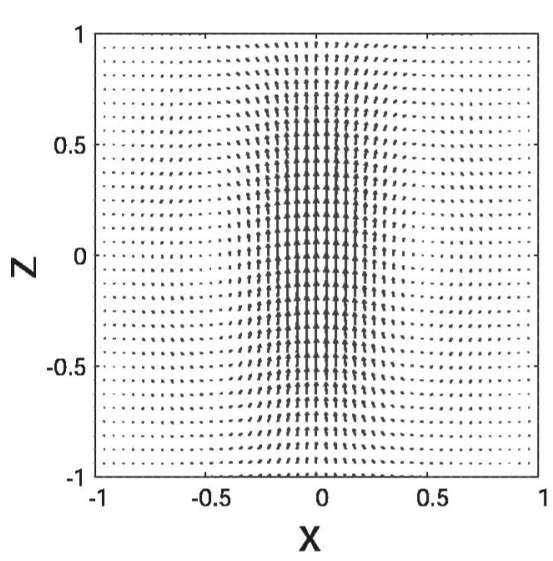

(c)

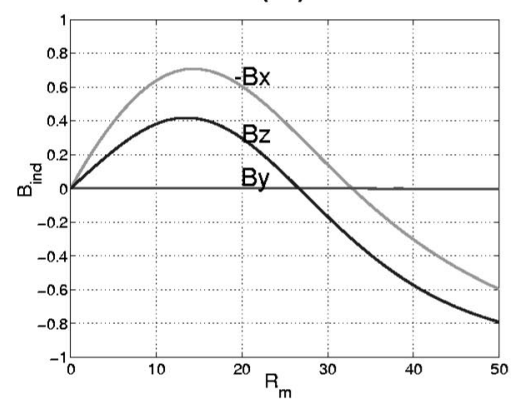

(a)

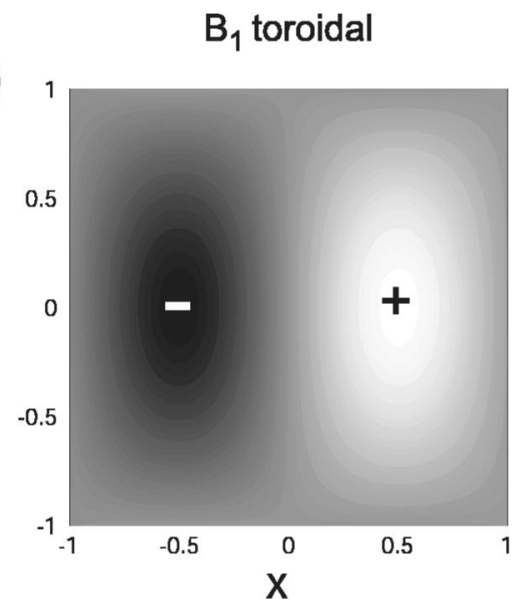

$\mathrm{B}_{2}$ toroidal

(b)

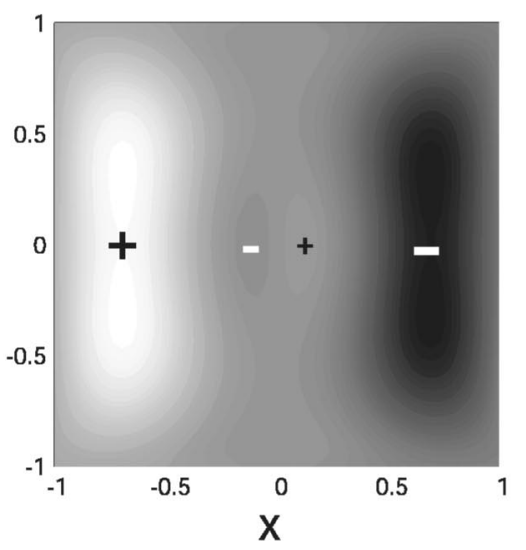

(d)

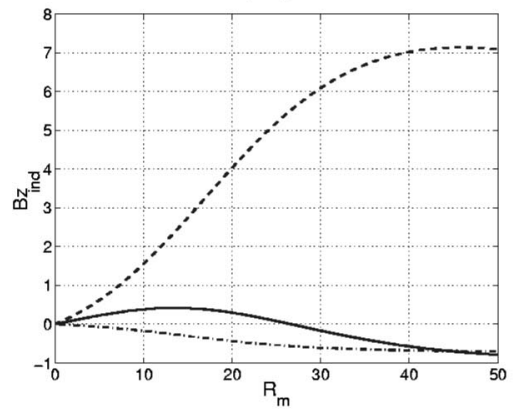

FIG. 5. Axial applied field. (a) Meridian cross section of the first order induced field. Poloidal component shown in left figure (arrows) and toroidal component in right figure, on a gray color scale; (b) magnetic field induced at second order; (c) variation with the $\mathrm{Rm}$ of the induced field at point ( $x=0, y=0.5, z=0)$ (Padé approximation is used); (d) evolution with $\mathrm{Rm}$ of the induced axial field at increasing radial distances $y=0.2$ (dashed), $y=0.5$ (solid), $y=0.8$ (dashdotted) from the axis. velocities. However, in the search for a dynamo mechanism, the poloidal velocity component is essential to give the flow a strong helicity and to generate a large scale " $\alpha$ " effect.

\section{HELICITY AND “ALPHA” EFFECT}

We consider now the SD case of a VK flow corresponding to the rotation of one disk in the experimental setup. We study its response to an external field applied perpendicularly to the cylinder axis (in this configuration axisymmetry is broken). We show that the main induction mechanism corresponds to the stretching and twisting of the imposed field lines (respectively by the axial and azimuthal velocities), as originally described by Parker. ${ }^{29}$ At second order, one ob- serves that the induced current has a strong component in the direction of the applied field; we thus call this effect an " $\alpha$ " effect, written with quotes to recall that although a result of flow helicity, it is not the $\alpha$ effect introduced in the meanfield hydrodynamics approach to model small scale contributions. ${ }^{30}$ As contributions at higher orders in $\mathrm{Rm}$ are computed, one observes again that induction is ultimately dominated by field expulsion due to the flow global rotation.

\section{A. First and second order contributions}

Let us begin with the first order contribution; it contains, when iterated, all the ingredients that lead to the " $\alpha$ " effect. Figure 7 (a) shows the electric potential $\phi_{1}$ in the flow vol- 


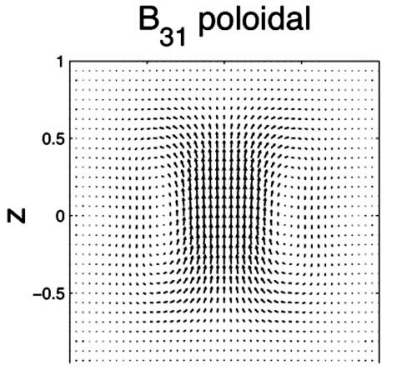

$\mathrm{B}_{32}$ poloidal

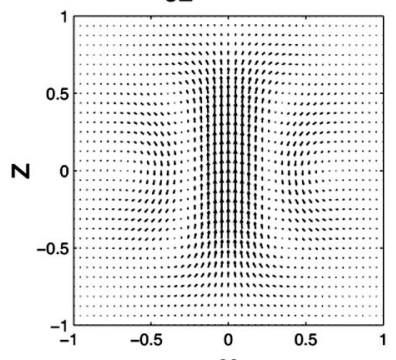

$\mathrm{B}_{33}$ poloidal

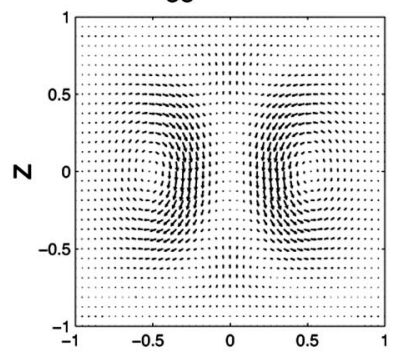

$\mathrm{B}_{34}$ poloidal

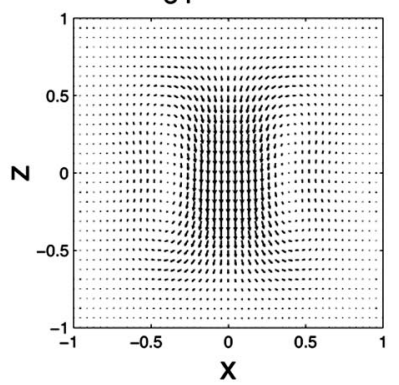

$B_{31}$ toroidal

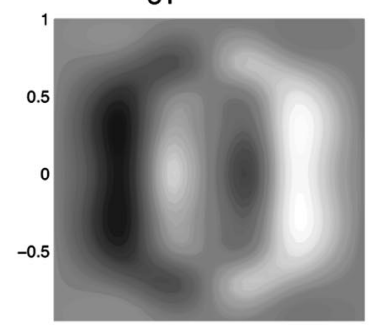

$\mathrm{B}_{32}$ toroidal

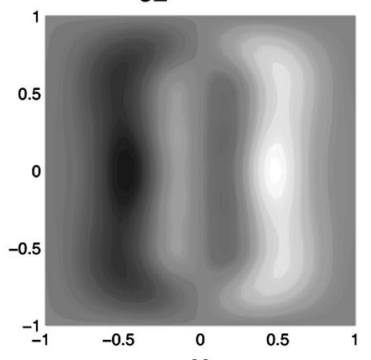

$\mathrm{B}_{33}$ toroidal
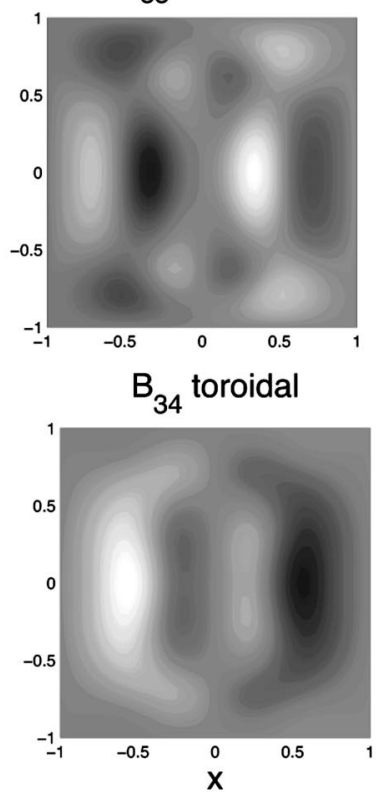

FIG. 6. Axial applied field. Induction iterations 31-34. Poloidal component shown in left figure (arrows) and toroidal component in right figure, on a gray color scale.

ume, for an applied field along the $x$ axis. As it is generated by the transverse part of the toroidal vorticity of the flow (one still has $\Delta \phi_{1}=\mathbf{B}_{0} \cdot \boldsymbol{\omega}$ and $\mathbf{B}_{0}$ is now perpendicular to the cylinder axis), it tends to create in the midplane a potential difference that is transverse and perpendicular to $\mathbf{B}_{0}$, thus aligned in the $y$ direction. However, its global structure is helicoidal, as shown by the shape of iso-potential surface $\left(\phi_{1}=0\right)$ in the figure. The induction e.m.f. $\mathbf{e}_{1}$ also has a $y$ component in the median plane (due to the axial velocity), but its sign is such that it opposes the electric potential (on the boundary they exactly compensate to ensure that the currents remain confined inside the cylinder). In contrast with the geometries described previously, the current distribution does not simply result from clearly identified potential or (a)

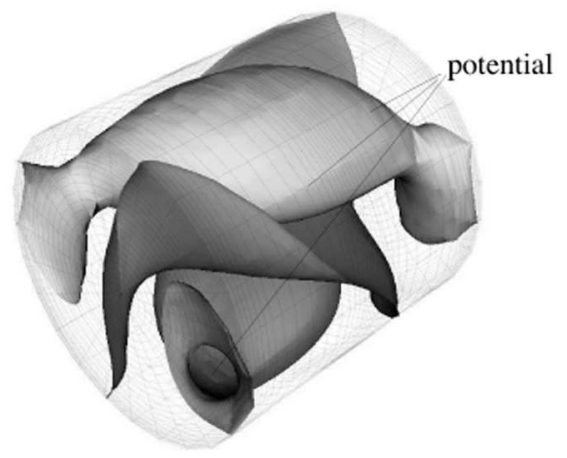

(b)

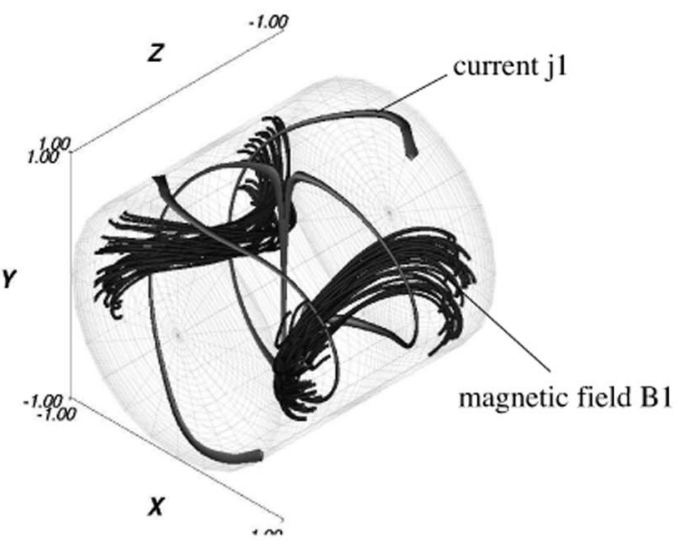

(c)

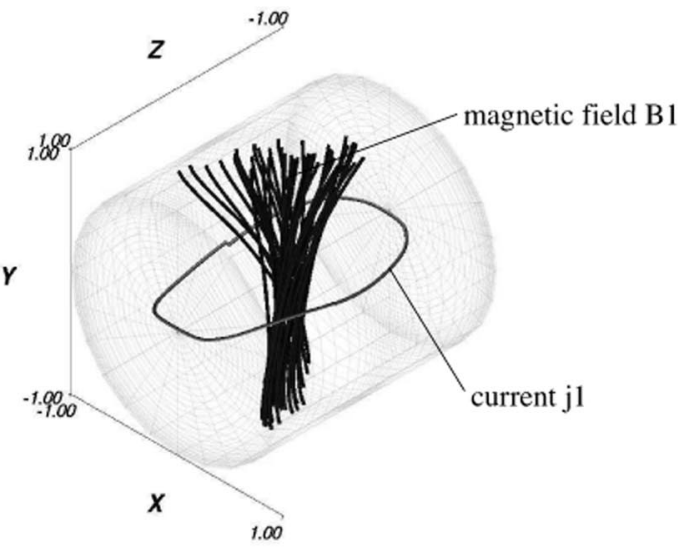

FIG. 7. Transverse applied field, (SD) geometry: first order response. (a) Potential, (b) current and axial induced field, (c) current and transverse induced field.

electromotive forces but stems from a delicate balance between the two effects. However, two essential current paths can be identified.

(i) Currents that flow in the transverse $y$ direction in the median plane, and with helical trajectories on either side of it-see Fig. 7(b). These currents generate an axial magnetic field component in the $x O z$ plane. Note that, whether of potential or electromotive origin, they are due to the poloidal part of the flow.

(ii) Currents that make a loop in the $x O z$ plane-Fig. 7 (c) - and thus generate an induced magnetic field along the $y$ axis. They are mainly generated by the toroidal part of the flow. These observation may be easier to understand from the 
(a)

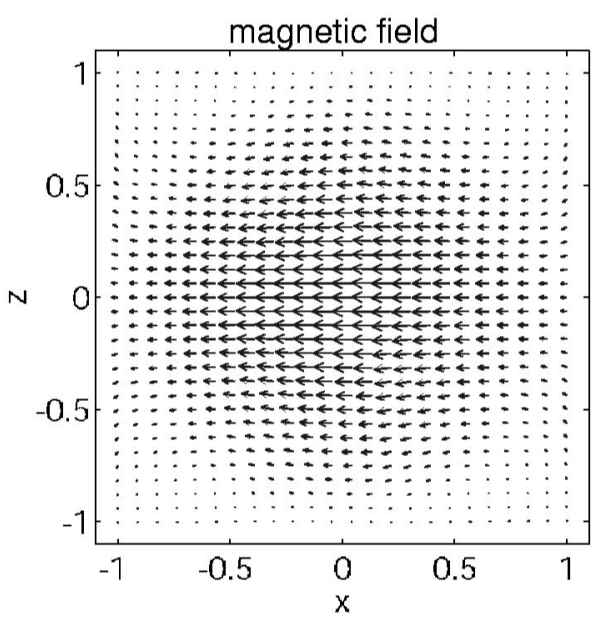

(c)

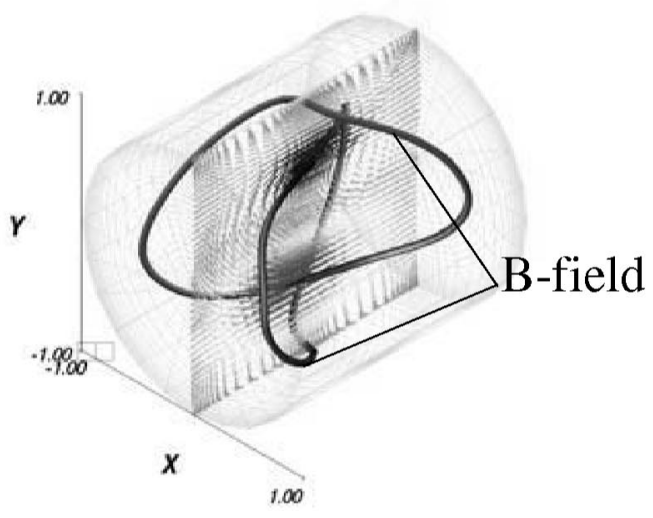

(b)

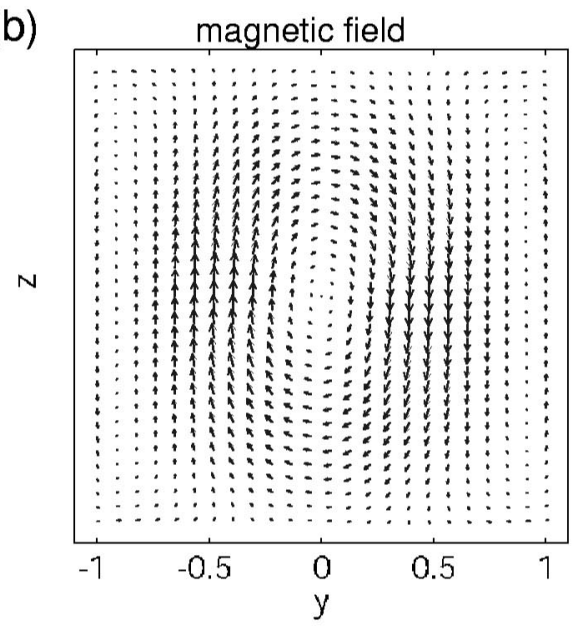

(d)

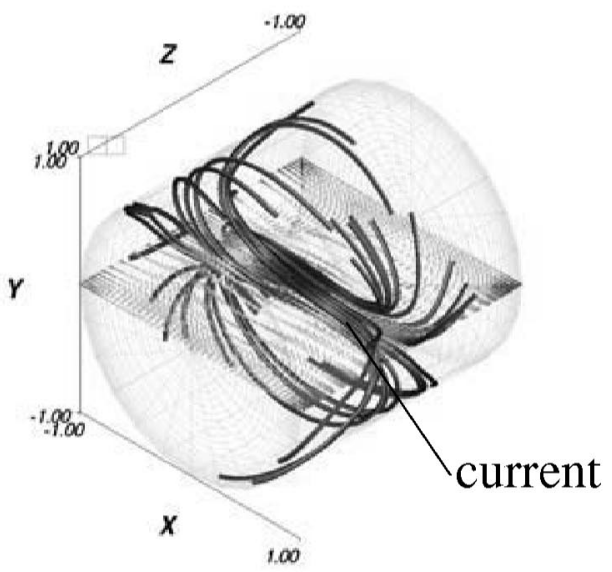

(e)

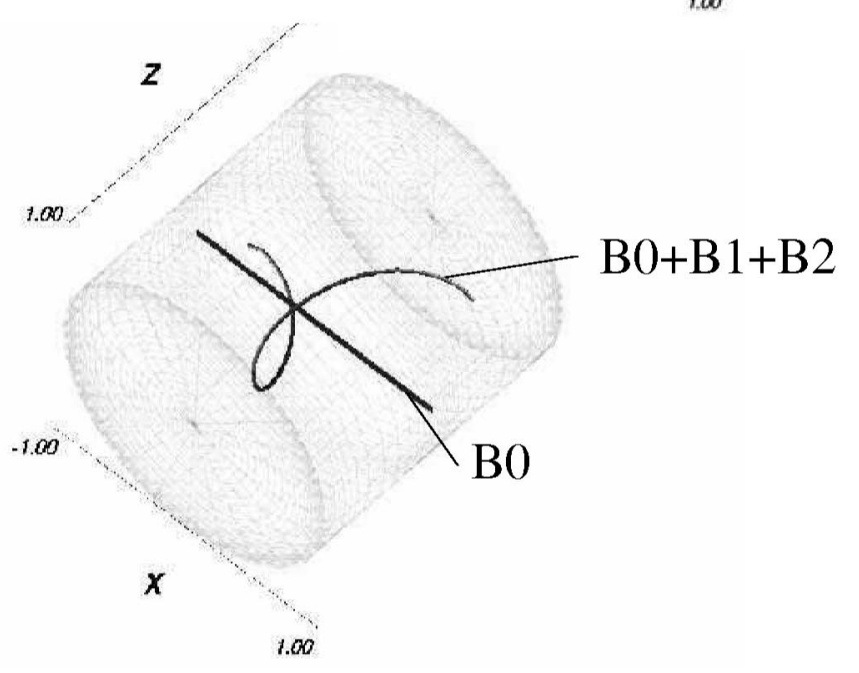

FIG. 8. Transverse applied field, (SD) geometry: second order induction. (a), (b) Projection of the second order induced field in the $x O z$ and $y O z$ planes; (c) magnetic field stream lines; (d) induced current; (e) net magnetic field up to second order; the stream line is selected to emphasize the " $\alpha$ "-effect mechanism.

point of view of advection/stretching of the imposed magnetic field lines. The poloidal flow deforms axially the applied field lines and creates an axial induced field, via velocity gradients such as $\partial_{r} u_{z}$. The toroidal flow tends to rotate the applied field ( $\partial_{r} u_{\theta}$ gradients) and thus to induce a magnetic field component in the transverse direction perpendicular to that of the applied field.

These processes are repeated at second order.

(i) The flow rotation acting on $B_{1, y}$ induces a component $B_{2, x}$ parallel but opposite to the applied field as shown in Fig. 8(a). This starts the expulsion of the transverse $\mathbf{B}_{0}$ by the axial vorticity of the flow, in the same manner as described in Sec. III.

(ii) The action of the axial pumping on $B_{1, y}$ and the action of the rotation on $B_{1, z}$ both lead to the induction of poloidal magnetic field lines $B_{2, z}$ in the $y O z$ plane [Figs. 8 (b) and 8(c)]. They are generated by the induction current $\mathbf{j}_{2}$ which has a strong component in the direction of the applied field $\mathbf{B}_{0}$, as shown in Fig. 8(d). One thus has $\mathbf{j}_{2} \propto \mathbf{B}_{0}$ in the 

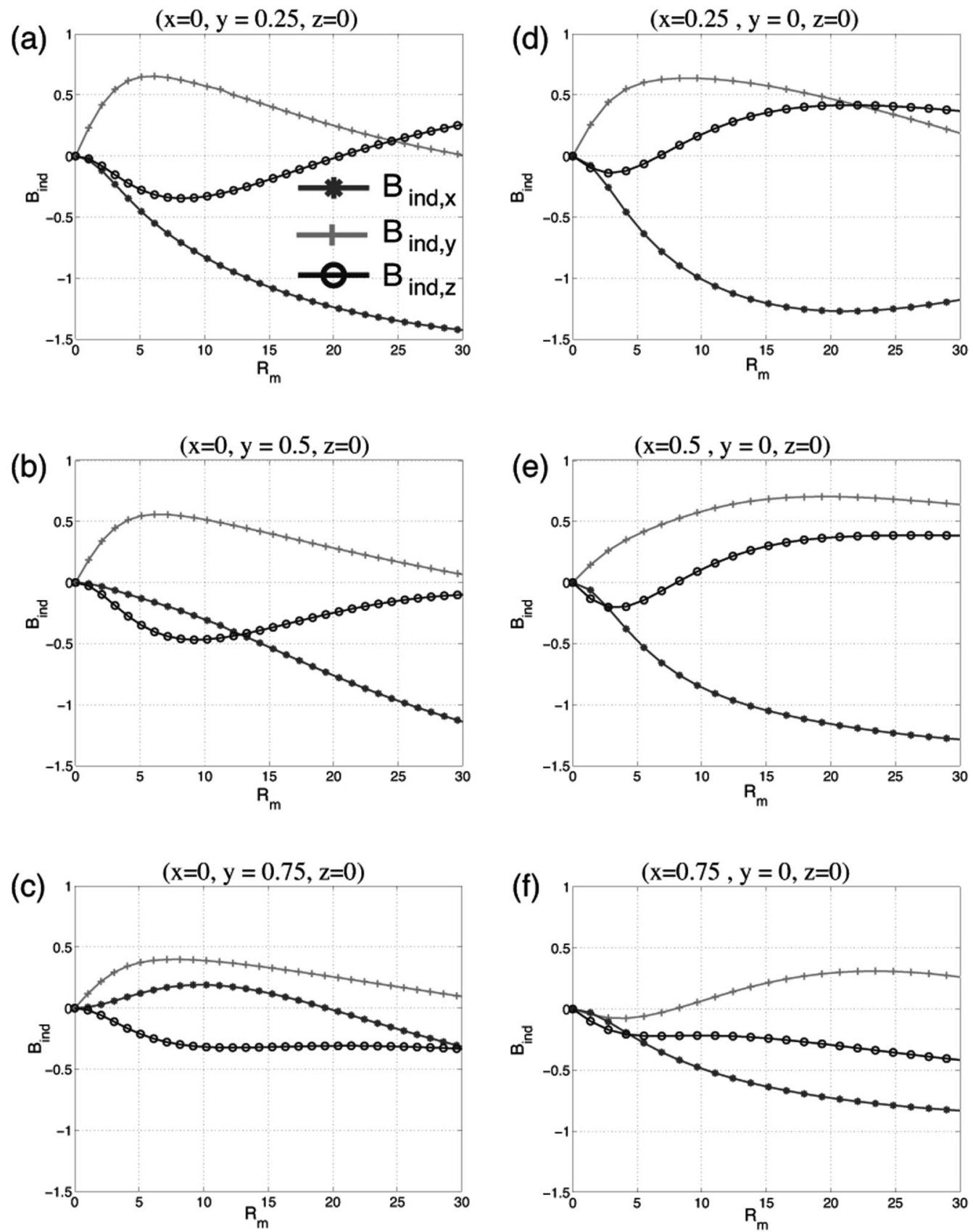

FIG. 9. Transverse applied field, (SD) geometry: local induction vs Rm. Points on the $y$ axis in the right figures, at $y=0.25,0.5,0.75$; and on the $x$ axis in the left figures, at $x=0.25,0.5,0.75$.

midplane of the flow-note that the currents are confined, so that near the walls of the cylinder they curl to loop inside the flow volume. $\mathbf{j}_{2}$ is thus parallel to $\mathbf{B}_{0}$ only in the center of the cell. The proportionality constant is quadratic in $\mathrm{Rm}$ and results from helicity of the flow: both the axial and rotational flow components have been needed to produce this second order mechanism. We call this effect an " $\alpha$ " effect, in analogy with the name introduced in the development of meanfield hydrodynamics by Rädler and Krause. ${ }^{30}$ However, here it is not a result of small scale contributions, but a macroscopic effect associated with the stretching and twisting of the applied field as originally proposed by Parker. ${ }^{29}$ Adding the first and second order induced fields to the applied one, it is actually possible to identify field lines that have the very topology proposed by Parker ${ }^{29}$-Fig. 8(e).

\section{B. Evolution with $\mathrm{Rm}$}

We first discuss the behavior at low to moderate magnetic Reynolds numbers, i.e., before field expulsion becomes dominant $(R m \leqslant 5)$. As the applied field has broken the axisymmetry, we treat induction separately for the $x O z$ and $y O z$ planes. We report the variations with $\mathrm{Rm}$ of the magnetic field computed at three different points in the flow volume. In the $y O z$ plane, Figs. 9(a), 9(b), and 9(c), the induction is dominated at low Rm by the " $\alpha$ " mechanism. The $x$ 

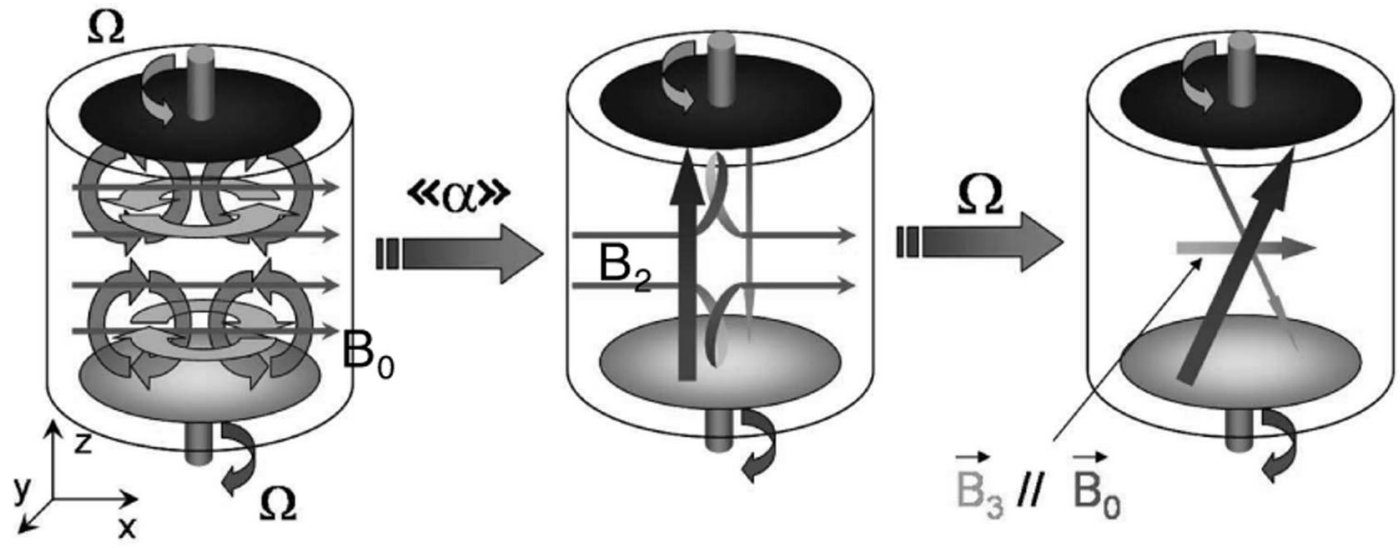

FIG. 10. "Alpha"-omega dynamo mechanism in the (DD) geometry: the applied transverse field (left) gives rise to the axial field displayed the middle figure - the " $\alpha$ " step; via the $\Omega$ effect, a third order induced field $\mathbf{B}_{3}$ is induced, parallel to $\mathbf{B}_{0}$.

and $z$ components vary quadratically with $R_{m}$, while the $y$ component is mostly linear in $\mathrm{Rm}$. The details of the $\mathbf{B}_{\text {ind }}(\mathrm{Rm})$ curves depend on the point of measurement. The $\alpha$ effect decreases with $r$ : compare the $x$ and $z$ components in Figs. 9(a), 9(b), and 9(c) where measurements are taken at distances $0.25,0.50$, and 0.75 from the rotation axis. In the $x O z$ plane [cf. Figs. 9(d), 9(e), and 9(f)] the $x$ component of the induced field displays a mostly quadratic variation with $\mathrm{Rm}$ (the expulsion effect) whereas the other two have a strong linear dependence. Qualitatively, these results are in very good agreement with the measurements made in flows having the same geometry. ${ }^{15,31}$

As the magnetic Reynolds number is further increased, the expulsion of the applied field from the core of the flow operates. Both the axial and toroidal vorticity components contribute to this expulsion. As a result the " $\alpha$ "-induction process is progressively cutoff from its source and its efficiency decreases. This evolution is also in good quantitative agreement with the measurements made in liquid sodium at Reynolds numbers up to 40: compare for example Fig. 5 in Ref. 31 and Fig. 9(b) here.

The predominance of expulsion at large Rm corresponds to a two-step negative loop-back mechanism at high orders of iteration. For example, we have observed that at large $n$, $\mathbf{B}_{n+2} \simeq \gamma \mathbf{B}_{n}$ with $\gamma=-3.63 \times 10^{-2}$. The value $\mathrm{Rm}^{*}$ $=1 / \sqrt{|\gamma|}=5.25$ gives very accurately the radius of convergence of the integer series (the curves in Fig. 9 have been computed using Padé approximants).

\section{AN ALPHA-OMEGA COOPERATIVE MECHANISM}

We consider again the (DD) flow geometry, i.e., the von Kármán flow generated by the counter-rotation of the driving disks. In each half of the cylinder the mean flow geometry is very similar to the (SD) geometry where it is known that the " $\alpha$ " effect generates an induced axial field from the applied transverse one. As the sign of the helicity is identical in both half cells, their contributions should add up. We thus expect in the (DD) case that when a transverse field (perpendicular to the cylinder axis) is applied, an axial field is induced in a two-step (quadratic) " $\alpha$ " mechanism. This induced field is distorted by the $\omega$ effect; in this process, a field component parallel to the initial applied field is generated, in a three-step (cubic) mechanism. This sequence-detailed in Fig. 10constitutes a positive induction loop, inducive of dynamo action.

\section{A. The alpha-omega induction process}

A transverse external field, $\mathbf{B}_{0}$ parallel to the $x$ axis is applied to the numerical (DD) flow. Induced fields are computed iteratively at $\mathrm{Rm}=1$, and shown in Fig. 11. The second order induced field $\mathbf{B}_{2}$-displayed in Fig. 11(a)-has an axial component in the $y \mathrm{Oz}$ plane. It is maximum in the regions of high helicity $(z= \pm 0.5)$, in the neighborhood of $y= \pm 0.5$ and has opposite direction on either side of the $x \mathrm{Oz}$ plane-as expected from the "alpha" mechanism described in the previous section. Note that, except for some magnetic diffusion, Fig. 11(a) is equivalent to twice the induction in Fig. 8(a). The third order induced field is shown in Fig. 11(b): one observes in the median plane the generation of a strong component of $\mathbf{B}_{3}$ along the $x$ axis ( $x O z$ plane). It corresponds to the torsion of the axial component of $\mathbf{B}_{2}$ by the differential rotation, via the $\omega$ effect. One thus obtains an induced field component aligned with the applied field $\mathbf{B}_{0}$, in agreement with the " $\alpha$ " $-\omega$ picture qualitatively described earlier.

Such a third order induction process can only be observed if the magnetic Reynolds number of the flow becomes large enough. Figure 12 shows the development of the induced magnetic field as $\mathrm{Rm}$ increases, for a point in the median plane. The direct series summation diverges for $\mathrm{Rm}>\mathrm{Rm}^{*}=8.5$ but was extrapolated by Padé approximants. In Fig. 12(a) one observes that at low Rm, the $x$ component of $\mathbf{B}_{\text {ind }}$ is negative and linear: it is due to the compression of the applied field by the radial flow (directed towards the rotation axis in the midplane). The tendency is reversed at higher Rm, and the $x$ component of $\mathbf{B}_{\text {ind }}$ becomes positive for $\mathrm{Rm}>17$.

A similar variation of the induced field in the case of an applied transverse field has been observed experimentally in the von Kármán sodium (VKS) experiment; in fact the evolution of $B_{\text {ind, } x}$ in Fig. 12(a) is quite similar to that in Fig. 5 of Ref. 19. 
(a)
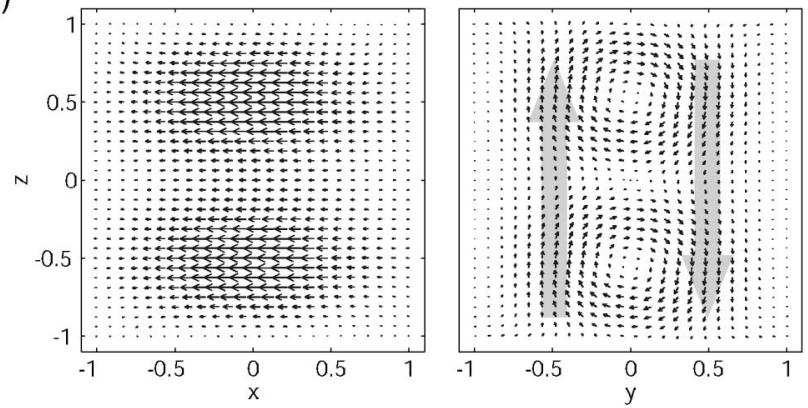

(b)
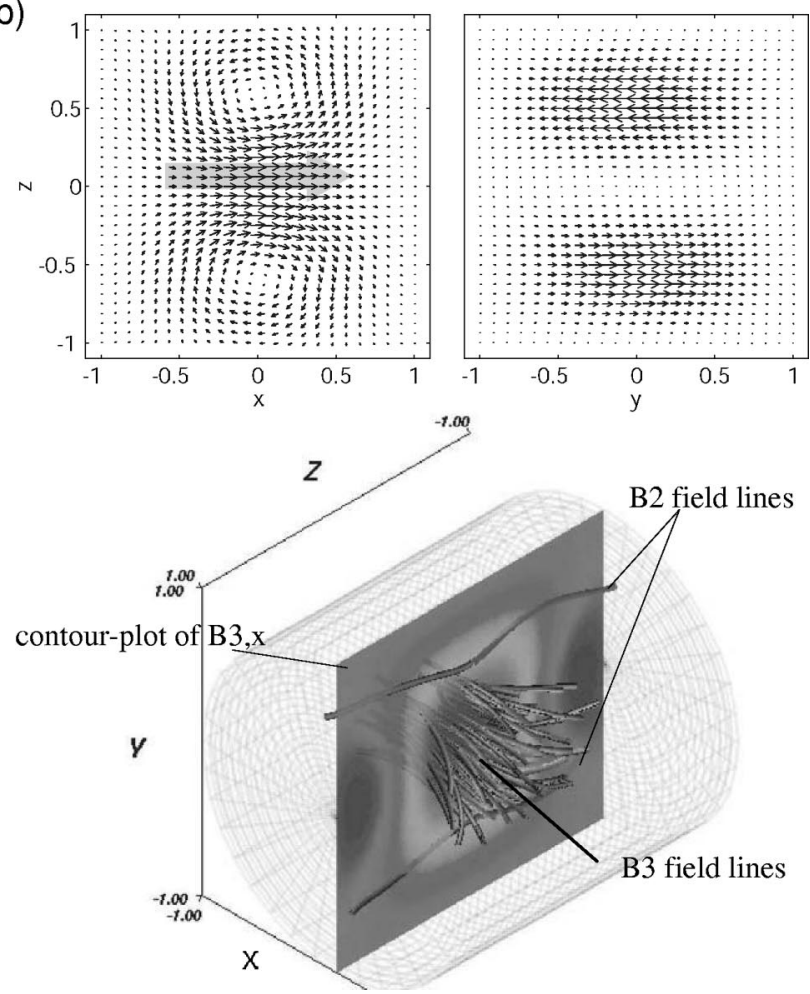

FIG. 11. " $\alpha$ " $-\omega$ induced fields in the (DD) geometry; $\mathbf{B}_{0}$ is applied along the $x$ axis. (a) Second order field $\mathbf{B}_{2}$; (b) third order field $\mathbf{B}_{3}$ (the gray arrows serve as an eye-guide); (c) three-dimensional view; selected second order and third order magnetic field lines are shown; the contour plot displays $B_{3, x}$.

\section{B. An alpha-omega dynamo?}

Starting from a initial transverse field, we have thus identified a three-step positive loop-back mechanism. It is tempting to associate this finding with the kinematic dynamos observed in von Kármán flows. ${ }^{11,12}$ It would provide a concrete mechanism for dynamo action-kinematic simulations address only the eventual existence of dynamo instability for a given flow, but do not explain how self generation is achieved. The knowledge of a dynamo mechanism is essential from the experimental point of view because it yields the possibility to optimize the dynamo cycle and thus lower the critical magnetic Reynolds number. However, the identification of von Kármán dynamos with the " $\alpha$ " $-\omega$ process described here should be made with some care.

Indeed, we have observed in our numerical study that when the iteration is carried to large orders, the dominant contribution comes from the expulsion of the magnetic field from the eddies in the flow. A two-step negative loop-back mechanism sets in. For example, at large $n \mathbf{B}_{n+2}$ is parallel and opposite to $\mathbf{B}_{n}$, with $\gamma=-1.39 \times 10^{-2}$. This gives a critical value $\mathrm{Rm}^{*}=1 / \sqrt{|\gamma|}=8.5$, in agreement with the observed critical value for the convergence of the numerical series.

It does not mean that the " $\alpha$ " $-\omega$ mechanism should be ruled out, but rather that it is not efficient enough in the flow geometry considered here to overcome the expulsion effect. To wit, let us return to Fig. 11(c): one may observe there that the generation of $B_{3, x}$ occurs predominantly in the center of the flow, where differential rotation is strongest. However, $B_{3, x}$ tends to vanish near $z= \pm 0.5$, in the regions of large vorticity where the " $\alpha$ " effect is strongest. The coupling between the two effects is thus far from being optimal and it may explain why it does not survive at the large $\mathrm{Rm}$.

The earlier considerations are consistent with the observations that dynamo action in von Kármán flows is extremely sensitive to the fine details of the flow geometry. For example, the ratio of the intensities of poloidal to toroidal velocity components ( $P / T$ ratio) is known to play a fundamental role. Duddley and James ${ }^{11}$ in their kinematic simula-
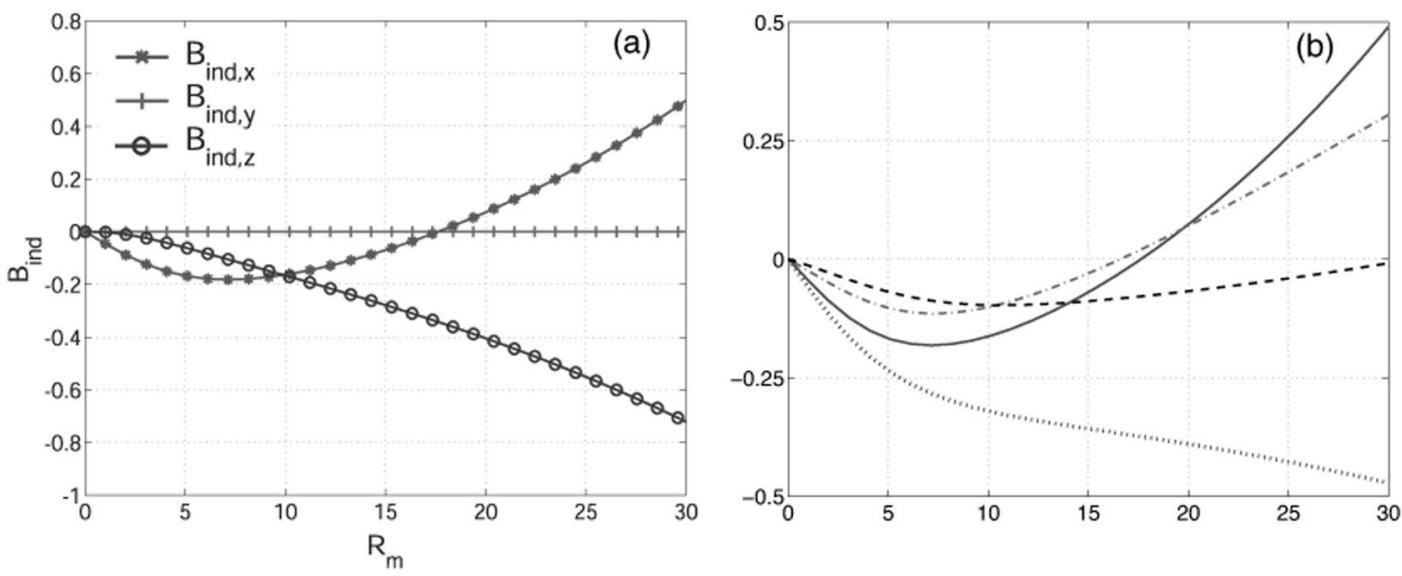

FIG. 12. "Alpha"-omega induced fields in the (DD) geometry; evolution of the induced field $\mathbf{B}_{\text {ind }}$ with the magnetic Reynolds number, computed using Padé approximants. (a) At $(x=0, y=0.5, z=0)$; (b) comparison of the induced $x$ component for various values of the poloidal to toroidal speeds ratio; dashed line: $P / T=0.2$; dotted-dashed line: $P / T=0.4$; solid line: $P / T=0.8$; dotted line: $P / T=1.6$. 

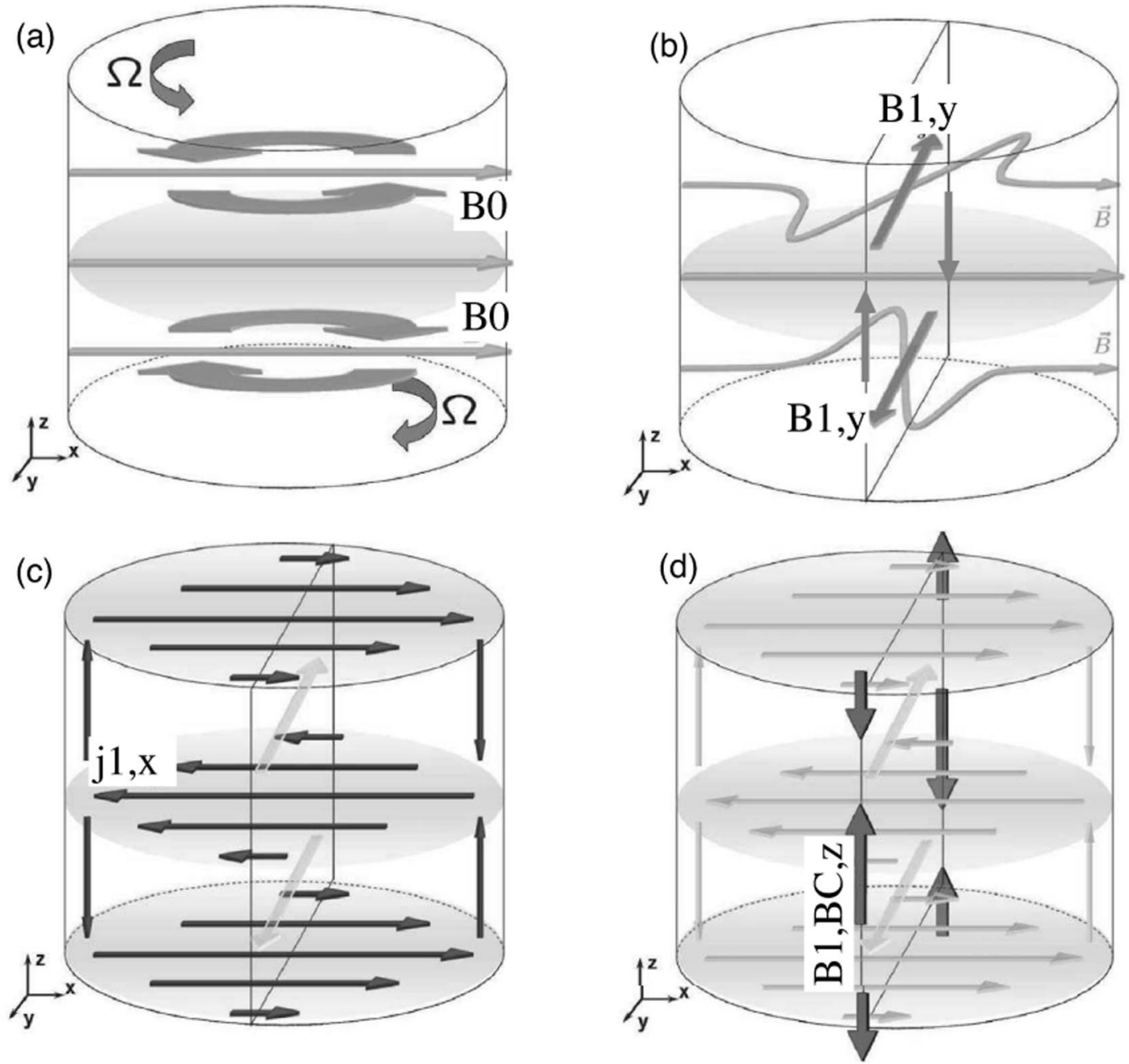

FIG. 13. Schematics of the BC effect for a transverse applied field $\mathbf{B}_{0}$ along the $x$ axis; (a) initial field and disks rotation; (b) the radial differential rotation generates a perpendicular component $B_{1, y}$; (c) corresponding induced current sheet $j_{1, x}$; (d) axial $B_{1, \mathrm{BC}, z}$ generated at the wall $(y= \pm R$ ), due to the discontinuity in the magnetic diffusivity.

tions in a spherical geometry have observed that selfgeneration occurs when $P / T \simeq 0.14$, but not when $P / T=0.1$ or $P / T=0.2$. If the toroidal velocity becomes too large, expulsion will surely dominate, but when it is too small the helicity and differential rotation are insufficient for an " $\alpha$ " $-\omega$ mechanism to develop. The results shown so far in our numerical study have been obtained for $P / T=0.8$, the optimal value as suggested by kinematic dynamo simulations. ${ }^{12}$ This value also corresponds to the optimal efficiency of the " $\alpha$ " $-\omega$ mechanism, as pointed out in Fig. 12(b).

\section{INFLUENCE OF BOUNDARY CONDITIONS}

One advantage of our iterative procedure is that various electrical boundary conditions can be considered. This is of importance because in real situations (natural or laboratory), the flow of conducting liquid is confined within walls whose electrical conductivity, magnetic permeability, thickness, etc., are important parameters in the induction process. The reason is that the magnetic diffusive length of liquid metals is often comparable to the integral length scale. For example, it has been shown that a conductive layer at rest surrounding the flow lowers the dynamo threshold in experimental configurations as tested in Riga and Karlsruhe. ${ }^{13}$ In this section, we first show that sizeable induction effects can result from the electrical boundary condition (BC effect), giving rise to induced magnetic field contributions which must be added to the bulk effects.

\section{A. BC effect for a transverse applied field}

Let us consider the case of the counter-rotating von Kármán geometry (DD flow), when an external field $\mathbf{B}_{0}$ is applied perpendicular to the rotation axis, in the $x$ direction. We start with the case of insulating boundary conditions at the flow wall and we will discuss later the generalization to other electrical conditions (note that it is the discontinuity of magnetic diffusivity that matters).

Let us first describe the $\mathrm{BC}$ effect in a qualitative manner. Because of the differential rotation, the $\mathbf{B}_{0}$ field lines are twisted to generate an induced component in the $y$ direction in the median plane-see Figs. 13(a) and 13(b). Actually, this is the first step in the generation of the " $\alpha$ " effect described 
(a)

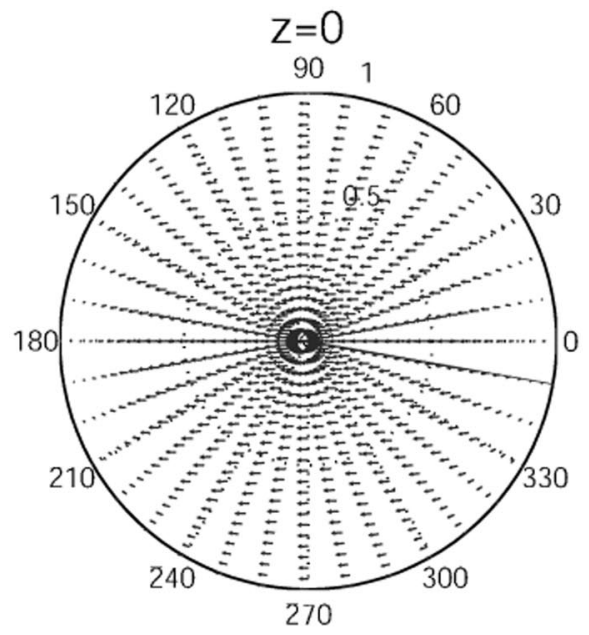

(c)

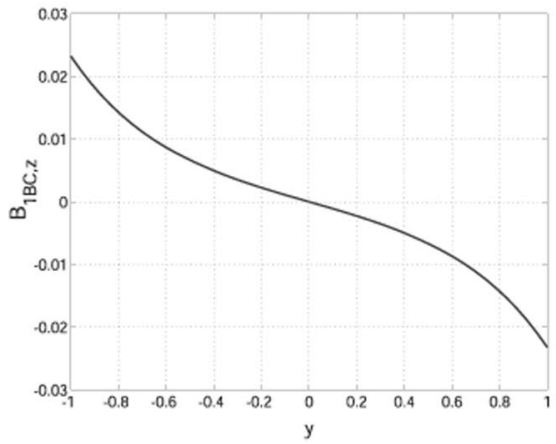

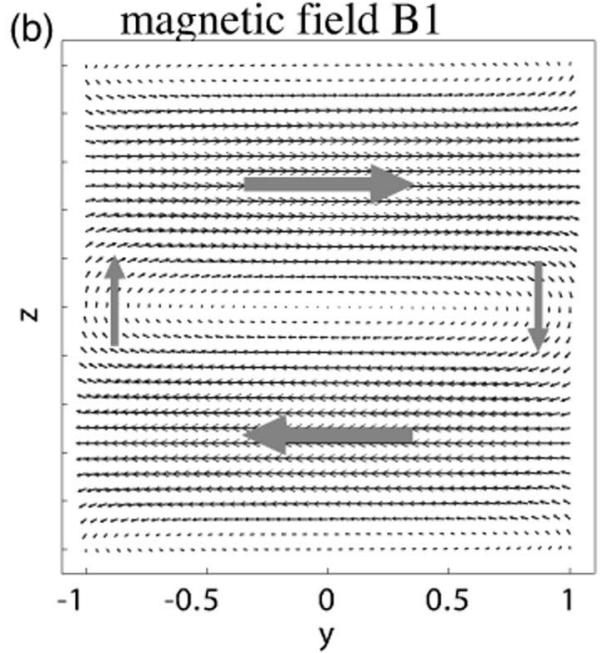

(d)

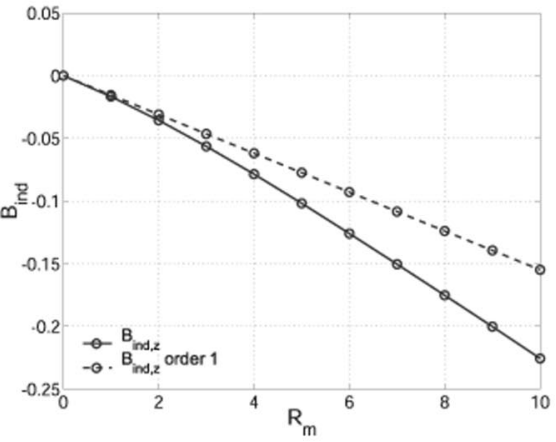

FIG. 14. BC effect, (DD) geometry: simulations (experimental flow field). (a) First order induced current $j_{1}$ in the $x O y$ at height $z=0$; (b) corresponding magnetic field in the $y O z$ plane; (c) profile of the BC-induced field $B_{1, \mathrm{BC}, z}(y)$ in median plane; (d) evolution with the magnetic Reynolds number at ( $x$ $=0, y=0.5, z=0$ ): first order contribution $B_{1, \mathrm{BC}, z}$ (dashed line) and net field $B_{\mathrm{BC}, z}$ (solid line).

in Sec. V. This field component $B_{1, y}$ is associated with a current layer $j_{1, x}$ that flows parallel to the applied field, and in opposite direction in the median plane. As the outside medium is electrically insulating, this current remains within the flow volume and is therefore tangent to the cylindrical vessel on the $y$ axis-see Fig. 13(c). At the wall, the discontinuity in $j_{1, x}$ creates an axial magnetic field $B_{1, \mathrm{BC}, z}$ outside of the flow volume, cf. Fig. 13(d). This field penetrates inside the flow because the diffusion length $(R / \sqrt{\mathrm{Rm}})$ is never small compared to the cylinder radius $R$. As a result, an axial magnetic field is created in the bulk of the flow, with its source in the boundary condition at the external wall. Its variation with the magnetic Reynolds number is essentially linear because the "source" $B_{1, y}$ is essentially produced from $\mathbf{B}_{0}$ by a first order induction mechanism. The magnetic field component $B_{1, \mathrm{BC}, z}$ can also be viewed as a necessity for the reconnection of the magnetic field lines of $\mathbf{B}_{1}$ at the lateral walls. We thus observe that this effect cannot exist independently of bulk induction effects; discontinuities of electrical conductivity alone do not generate an induced field!

The schematic picture described above is supported by the numerical simulation. Figure 14(a) shows the induced current in the $x O y$ plane, which lies parallel to the applied field in the middle of the cell. It is therefore tangent to the cylinder walls near $y= \pm R$. At these points the current discontinuity generates the induced field $B_{1, \mathrm{BC}, z}$ as shown in Fig. 14(b). The evolution of this component inside the flow volume is shown in Fig. 14(c): as expected it is maximum at the wall and decreases slowly away from it (its value is only halved at $y=0.7)$. The $\mathrm{BC}$ effect is essentially linear as can be observed in Fig. 14(d) where the evolution of the induced magnetic field with the Reynolds number at point $(x=0, y$ $=0.5, z=0$ ) is reported: the dashed line shows the contribution of the first order induction alone, while the solid line has been computed summing all orders.

Finally, we should comment upon the symmetries of the field induced by this boundary effect: even symmetry with respect to the $x O y$ plane and odd symmetry with respect to the $x O z$ plane. No linear bulk induction mechanism has the same symmetries. Indeed, in the bulk, the axial field at first order is generated by the radial gradient of the axial flow (a $B_{0} \cos \theta \partial_{r} u_{z}$ term in the induction equation), a term which is odd with respect to the $x O y$ plane. Thus there is no linear bulk effect that can produce a field which is even in symmetry with respect to the $x O y$ plane. However, the quadratic " $\alpha$ " effect has the same symmetries as the BC effect. In actual experimental situations, both effects will occur simultaneously; but if the induction is observed to vary mainly linearly with the magnetic Reynolds number one may conclude that the effect of boundary conditions are dominant over the " $\alpha$ " effect—as in the VKS measurements. ${ }^{17}$

We now discuss further some of the properties of this induction effect due to boundary conditions. As noted in Sec. II A, inhomogeneities in the magnetic diffusivity can be in- 


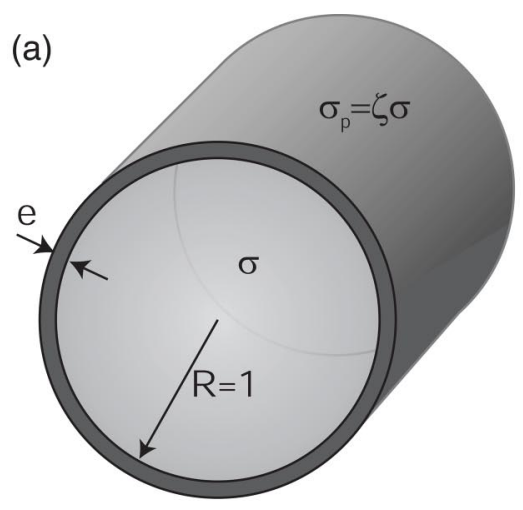

(c)

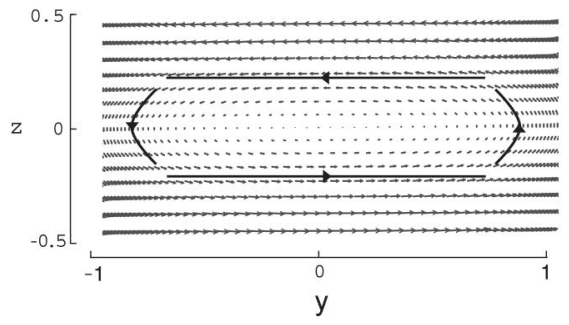

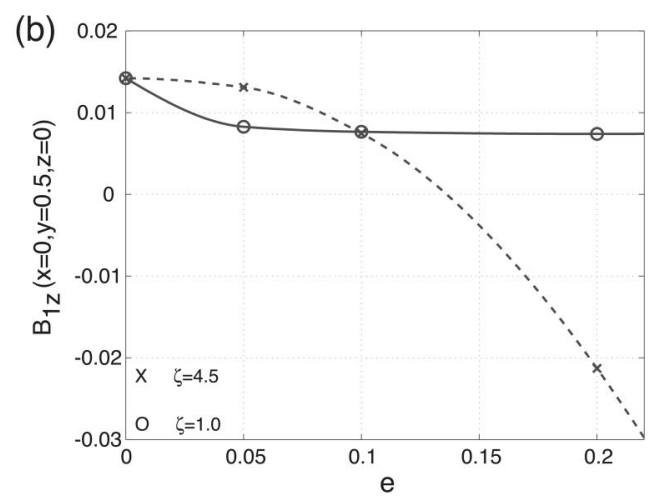

(d)

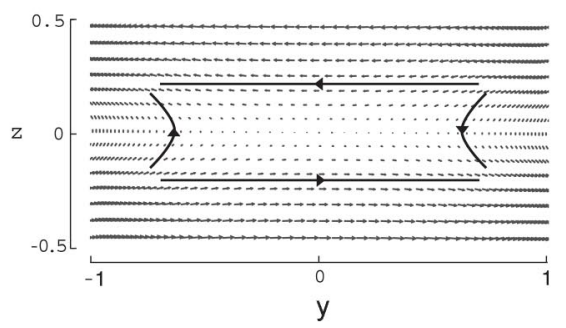

FIG. 15. BC effect, (DD) geometry (experimental flow field): influence of the vessel thickness and electrical conductivity. (a) Sketch of the vessel geometry; (b) evolution of the first order BC axial induced field, with the thickness of the vessel (the dashed and solid lines are only meant as guide to the eye); (c) and (d) magnetic field topology in the case of a change of the sign of the discontinuity of the electrical conductivity at the wall (left: $\xi<1$; right $\xi>1$ ).

cluded in the induction equation [Eq. (1)]. To lowest order, as we have seen that the $\mathrm{BC}$ effect is mostly linear, it can be written as

$$
\lambda \Delta \mathbf{B}_{1}=-\nabla \times\left(\mathbf{u} \times \mathbf{B}_{0}\right)-[\lambda]_{(S)} \delta_{(S)}\left(\nabla \times \mathbf{B}_{1}\right) \times \mathbf{n},
$$

where $S$ is the surface over which the magnetic diffusivity discontinuity occurs, $\mathbf{n}$ the outgoing normal, $[\lambda]_{(S)}$ is the jump in magnetic diffusivity and $\delta_{(S)}$ a Dirac distribution null everywhere except on $S$. This is a closed form in which $\mathbf{B}_{1}$ results both from the bulk and boundary effects. It is possible to separate these contributions because the boundary effects stem from the bulk induction. First, we isolate the specific induction source term that generates the BC effect and express it in terms of the induced current $\mathbf{j}_{1}$ :

$$
\mathbf{I}_{1, \mathrm{BC}}=-[\lambda]_{(S)} \delta_{(S)} \mathbf{j}_{1} \times \mathbf{n} .
$$

This current is mainly generated by the bulk induction, so that to lowest order it verifies the Poisson equation

$$
\Delta \mathbf{j}_{1}=-\frac{1}{\mu_{0} \lambda}\left(\mathbf{B}_{0} \cdot \boldsymbol{\nabla}\right) \boldsymbol{\omega}
$$

where $\boldsymbol{\omega}=\boldsymbol{\nabla} \times \mathbf{u}$ is the flow vorticity and we have assumed that the applied field $\mathbf{B}_{0}$ is homogeneous. Using this formulation one expects the $\mathrm{BC}$ effect to be quite sensitive to the precise structure (vorticity distribution) of the flow. It is indeed the case: the BC effect is weak in the numerical model field used in our study, but very strong in actual experiments. ${ }^{15,18}$ The reason is that experimental flows have a stronger radial vorticity gradient in the vicinity of the cylinder lateral walls ${ }^{25}$ and thus promote a stronger induction according to Eqs. (31)-(33). This is why in order to enhance the BC effect, the curves in Fig. 14 have been computed using experimental flow profiles.

\section{B. When the external walls are a thin conducting shell}

As discussed earlier the source term of the BC effect [Eq. (31)] is proportional to the jump in magnetic diffusivity at the wall. It has opposite effects depending on whether the outside medium has a higher or lower electrical conductivity than the fluid. An interesting situation occurs when the flow walls are made of a shell of material with a higher conductivity, surrounded by an insulating medium. For example, this would be the case for a sodium flow enclosed in a copper vessel, surrounded by air.

We consider the case of a von Kármán flow enclosed in a cylindrical vessel with thickness $e_{p}$ and conductivity $\sigma_{p}$ $=\zeta \sigma$-cf. Fig. 15(a). Note that in such a geometry, the equation for the electric potential (8) must take into account the inhomogeneity of the magnetic diffusivity and thus be replaced by

$$
\Delta \phi_{k+1}-\nabla \lambda \cdot \nabla_{\phi k+1}=\boldsymbol{\nabla} \times\left(\mathbf{u} \times \mathbf{B}_{k}\right)-\nabla \lambda \cdot\left(\mathbf{u} \times \mathbf{B}_{k}\right),
$$

with the condition $(\boldsymbol{\nabla} \phi)_{n}=0$ at the outer insulating wall.

We concentrate on $\zeta=1$ (the walls have an electrical conductivity equal to that of the fluid) and $\zeta=4.5$ (the ratio of electrical conductivities of copper and liquid sodium). In each case the variation of the axial field induced by the $\mathrm{BC}$ effect with the thickness of the vessel is shown in Fig. 15(b) [at point $(x=0, y=0.5, z=0)]$. 
(1) When $\zeta=1$, the boundary effect tends first to decrease as the thickness of the vessel is increased, up to a thickness $e_{p}$ equal to approximately $5 \%$ of the radius of the cylinder. Then for $e_{p}>0.05 R$, the magnitude of the BCinduced field is roughly constant at a value equal to $60 \%$ of its amplitude at $e_{p}=0$ (perfectly insulating outside medium).

(2) When $\zeta=4.5$, the BC-induced field remains at a constant level for $e_{p} \leqslant 0.05 R$, but then decreases and changes its sign for $e_{p}>0.13 R$. For higher values of $e_{p}$ the induced field remains negative, as expected from a direct calculation with a full external medium with electrical conductivity larger than that of the fluid.

We have already mentioned that the $\mathrm{BC}$ effect is associated with the reconnection of the bulk magnetic field $\mathbf{B}_{1}$ on either side of the median plane. We note here that the change of sign of the induced field corresponds to different reconnection patterns, as shown in Figs. 15(c) and 15(d) and also observed in laboratory plasma experiments. ${ }^{32}$

\section{CONCLUDING REMARKS}

In this paper we have presented a new approach of induction in flows of conducting liquids, in which the contributions are computed iteratively. Its numerical implementation is simple and gives access to the complete set of electromagnetic variables involved in the induction: electric potential, currents, and magnetic field. Realistic boundary conditions can be considered. This scheme proves to be very convenient to identify how induction mechanisms develop as the magnetic Reynolds number of the flow increases, and how they are related to the topology of the flow field. Investigations of geometries that are relevant to geophysical situations are currently underway. In addition, the method which is at present restricted to stationary flows, could be readily extended for simple time-periodic flows.

From a practical point of view our approach has enabled us a clear identification of important induction mechanisms in von Kármán flows. When compared to experimental measurements, our approach has lead to a very good quantitative agreement, without any adjustable parameter. ${ }^{33}$ The origin and development of the " $\alpha$ " and $\omega$ effects have been described in detail, as well as their interaction with expulsion which seems to dominate when the magnetic Reynolds number becomes large. We have also pointed out that boundary conditions may be essential in the induction process, particularly when the magnetic dissipation length is not small compared to the flow integral scale (as is the case of most natural/experimental situations). As an example, we have shown that an external layer of conducting material behaves very differently than an external infinite conducting medium.

In regards to the dynamo generation, it is viewed here as a positive loop-back mechanism in the iteration of the induction process. A dynamo is identified if the operator $\mathcal{G}(\mathrm{Rm}$ independent) has a positive eigenvalue. The neutral mode then has the geometry of the associated eigenvector. As explained, this approach has a firm link with the usual kinematic dynamo framework. In the case of von Kármán flows, we have identified a possible $\alpha-\omega$ mechanism, responsible for the self generation observed in kinematic simulations. ${ }^{11,12}$ However, we have also observed that at the highest orders of iteration, a negative feedback loops sets in, associated with the expulsion of the applied field by the large scale eddies of the flow. It shows that expulsion is the most efficient induction mechanism at large $\mathrm{Rm}$, and that it may mask other processes. In fact, the study of the evolution of the mean induced field in the presence of an externally applied magnetic field may not allow a direct identification of the dynamo threshold.

\section{ACKNOWLEDGMENTS}

The authors thank all members of the VKS team (A. Chiffaudel, F. Daviaud, S. Fauve, L. Marié, F. Pétrélis, F. Ravelet, R. Volk) with whom we have had numerous fruitful discussions. They are particularly indebted to F. Ravelet for making his experimental flow fields available. They also acknowledge stimulating discussions with P. Frick.

${ }^{1}$ J. Larmor, "How could a rotating body such as the sun become a magnet," Report of the 87th Meeting of the British Association for the Advancement of Science (John Murray, London, 1919), pp. 159-160.

${ }^{2}$ F. J. Lowes and I. Wilkinson, "Geomagnetic dynamo: A laboratory model," Nature (London) 198, 1158 (1963).

${ }^{3}$ R. Steglitz and U. Müller, "Experimental demonstration of a homogeneous two-scale dynamo," Phys. Fluids 13, 561 (2001).

${ }^{4}$ A. Gailitis, O. Lielausis, S. Dement'ev, E. Platacis, A. Cifersons, G. Gerbeth, T. Gundrum, F. Stefani, M. Christen, H. Hänel, and G. Will, "Detection of a flow induced magnetic field eigenmode in the Riga dynamo facility," Phys. Rev. Lett. 84, 4365 (2000).

${ }^{5}$ G. O. Roberts, "Dynamo action of fluid motions with two-dimensional periodicity," Philos. Trans. R. Soc. London, Ser. A 271, 411 (1972).

${ }^{6}$ Yu. B. Ponomarenko, "On the theory of the hydromagnetic dynamo," J. Appl. Mech. Tech. Phys. 14, 775 (1973).

${ }^{7}$ A. Tilgner, "A kinematic dynamo with a small scale velocity field," Phys. Lett. A 226, 75 (1997).

${ }^{8}$ A. Gailitis, "Project of a liquid sodium MHD dynamo experiment," Magnetohydrodynamics (N.Y.) 1, 63 (1996)

${ }^{9}$ See, for example, L. Marié, F. Pétrélis, M. Bourgoin, J. Burguete, A. Chiffaudel, F. Daviaud, S. Fauve, P. Odier, and J.-F. Pinton, "Open questions about homogeneous fluid dynamos: The VKS experiment," Magnetohydrodynamics 38, 163 (2002); W. L. Shew, D. R. Sisan, and D. P. Lathrop, "Mechanically forced and thermally driven flows in liquid sodium," ibid. 38, 121 (2002), and references therein.

${ }^{10} \mathrm{H}$. K. Moffatt, Magnetic Field Generation in Electrically Conducting Fluids (Cambridge University Press, Cambridge, 1978).

${ }^{11}$ N. L. Dudley and R. W. James, "Time-dependent kinematic dynamos with stationary flows," Proc. R. Soc. London, Ser. A 425, 407 (1989).

${ }^{12}$ L. Marié, J. Burguete, F. Daviaud, and J. Léorat, "Numerical study of homogeneous dynamo based on experimental von Kármán type flows," Eur. Phys. J. B 33, 469 (2003).

${ }^{13}$ R. Avalos-Zuniga, F. Plunian, and A. Gailitis, "Influence of electromagnetic boundary conditions onto the onset of dynamo action in laboratory experiments," Phys. Rev. E 68, 066307 (2003).

${ }^{14}$ P. Odier, J.-F. Pinton, and S. Fauve, "Advection of a magnetic field by a turbulent swirling flow,” Phys. Rev. E 58, 7397 (1998).

${ }^{15}$ M. Bourgoin, R. Volk, P. Frick, S. Kripechenko, P. Odier, and J.-F. Pinton, "Induction mechanisms in a von Kármán swirling flow of liquid gallium," Magnetohydrodynamics 40, 13 (2004).

${ }^{16}$ N. L. Peffley, A. B. Cawthrone, and D. P. Lathrop, "Toward a selfgenerating magnetic dynamo: The role of turbulence," Phys. Rev. E 61, 5287 (2000).

${ }^{17}$ L. Marié, J. Burguete, A. Chiffaudel, F. Daviaud, E. Ericher, C. Gasquet, F. Pétrélis, S. Fauve, M. Bourgoin, M. Moulin, P. Odier, J.-F. Pinton, A. Guigon, J.-B. Luciani, F. Namer, and J. Léorat, "MHD in von Kármán swirling flows," in Dynamo and Dynamics: A Mathematical Challenge, Proceedings of the NATO Advanced Research Workshop, Cargèse, France, 
21-26 August, 2000, NATO Science Series II Vol. 26, edited by P. Chossat, D. Armbruster, and I. Oprea (Kluwer Academic, Dordrecht, 2001).

${ }^{18}$ M. Bourgoin, L. Marié, F. Petrelis, J. Burguete, A. Chiffaudel, F. Daviaud, S. Fauve, P. Odier, and J.-F. Pinton, "MHD measurements in the von Karman sodium experiment," Phys. Fluids 14, 3046 (2002).

${ }^{19}$ L. Marié, F. Petrelis, M. Bourgoin, J. Burguete, A. Chiffaudel, F. Daviaud, S. Fauve, P. Odier, and J.-F. Pinton, "Open questions about homogeneous fluid dynamos; the VKS experiment," Magnetohydrodynamics 38, 163 (2002).

${ }^{20}$ A. Martin, P. Odier, J.-F. Pinton, and S. Fauve, "Effective permeability in a binary flow of liquid gallium and iron beads," Eur. Phys. J. B 18, 337 (2000).

${ }^{21}$ W. H. Press, S. A. Teukolsky, W. T. Vetterling, and B. P. Flannery, Numerical Recipes (Cambridge University Press, Cambridge, 1988).

${ }^{22} \mathrm{G}$. A. Baker, "Application of the Padé approximants method to the investigation of some magnetic properties of the Ising model," Phys. Rev. 124, 768 (1961).

${ }^{23}$ Y. Ponty, H. Politano, and J.-F. Pinton, "Simulation of induction at low magnetic Prandtl number," Phys. Rev. Lett. 92, 144503 (2004).

${ }^{24}$ Overture library, "Object-oriented tools for solving PDEs in complex geometries," http://www.llnl.gov/CASC/Overture
${ }^{25}$ F. Ravelet, M.S. thesis, Pierre \& Marie Curie University, 2002.

${ }^{26}$ N. O. Weiss, "The expulsion of magnetic flux by eddies," Proc. R. Soc. London, Ser. A 293, 310 (1966).

${ }^{27}$ P. Odier, J.-F. Pinton, and S. Fauve, "Magnetic induction by coherent vortex motion," Eur. Phys. J. B 16, 373 (2000).

${ }^{28}$ R. L. Parker, "Reconnexion of lines of force in rotating spheres and cylinders," Proc. R. Soc. London, Ser. A 291, 60 (1966).

${ }^{29}$ E. N. Parker, "Hydromagnetic dynamo models," Astrophys. J. 163, 255 (1955).

${ }^{30}$ F. Krause and K.-H. Rädler, Mean Field Magnetohydrodynamics and Dynamo Theory (Pergamon, New York, 1980).

${ }^{31}$ F. Pétrélis, M. Bourgoin, L. Marié, J. Burquete, A. Chiffaudel, F. Daviaud, S. Fauve, P. Odier, and J.-F. Pinton, "Nonlinear magnetic induction by helical motion in a liquid sodium turbulent flow," Phys. Rev. Lett. 90, 174501 (2003).

${ }^{32}$ M. Yamada, H. Ji, S. Hsu, T. Carter, R. Kulsrud, Y. Ono, and F. Perkins, "Identification of Y-shaped and O-shaped diffusion regions during magnetic reconnection in a laboratory plasma," Phys. Rev. Lett. 78, 3117 (1997).

${ }^{33}$ M. Bourgoin, Ph.D. thesis, Ecole Normale Supérieure de Lyon, 2003. 